\title{
UN SIGLO Y MEDIO DE VELOCIDAD DE CIRCULACIÓN DEL DINERO EN ESPAÑA: ESTIMACIÓN Y DETERMINANTES*
}

\author{
ALBERT CARRERAS \\ Universitat Pompeu Fabra ${ }^{a}$ \\ CONCEPCIÓN GARCÍA-IGLESIAS \\ Universidad de Helsinki ${ }^{\text {b }}$ \\ JUHA KILPONEN \\ Banco de Finlandia ${ }^{\mathrm{c}}$
}

\section{RESUMEN}

Presentamos una serie temporal de la velocidad de circulación del dinero en España usando la M2 y explicamos su comportamiento en el siglo y medio que va de 1850 a 2000 . La velocidad de circulación del dinero en España presenta un declive secular hasta 1973 y apunta la forma de U en el último cuarto del siglo Xx. Existe un retraso en los cambios de tendencia con respecto a otros países industrializados, explica-

* Agradecemos a Sebastián Coll, Pablo Martín Aceña y M. Ángeles Pons, Leandro Prados de la Escosura y Xavier Tafunell su generosidad al facilitarnos y permitirnos usar investigaciones suyas inéditas. La investigación se ha realizado con ayuda del proyecto de investigación SEC2000-1084, BEC2003-00190 y de las ayudas a la investigación de la UPF. Nos han sido de mucha utilidad los comentarios recibidos en la presentación de versiones preliminares en Buenos Aires (XIII Congreso de la Asociación Internacional de Historia Económica, agosto 2002), en la Universitat Pompeu Fabra (seminario de Historia Económica, octubre 2002) y en Iberometrics-I (First Iberian Cliometrics Workshop, Lisboa, marzo 2003). Agradecemos particularmente los comentarios de nuestro «discussant» en Lisboa -Álvaro Aguiar- y de Pablo Martín Aceña. Dos «referees» anónimos de la Revista de Historia Económica nos han ayudado a corregir y mejorar significativamente el primer manuscrito. Evidentemente, todos los errores son de nuestra exclusiva responsabilidad.

a Departamento de Economía y Empresa. Ramón Trias Fargas, 25-27. E-08005 Barcelona, España. albert.carreras@upf.edu.

b Departamento de Historia Económica y Social. PO Box 54, FIN-00014 Helsinki, Finlandia.conchita.garcia-iglesias@helsinki.fi.

c Departamento de Política Monetaria e Investigación. PO Box 160, FIN-00101 Helsinki, Finlandia.juha.kilponen@bof.fi. 
ble por el atraso económico español. Estimamos los determinantes de la velocidad de circulación de dinero - la inversa de la demanda de dinero- para todo el período de 1850 a 1997. Un análisis de cointegración y un mecanismo de correción de errores nos permiten afirmar que existe una función estable para todo el período donde las variables institucionales son importantes para explicar el comportamiento de la velocidad en España en el largo plazo.

Palabras clave: Velocidad de circulación del dinero, Demanda de dinero, España, 1850-2000

\begin{abstract}
We present the yearly evolution of the Spanish income velocity of money (M2) and we explain its behavior from 1850 to 2000. The Spanish income velocity of money displays a downward trend until 1973 and shows the expected U-shaped pattern at the end of the twentieth century. An international comparison shows how the Spanish trend change occurs with a lag due to the late industrialization. We estimate the determinants of the velocity - the inverse of the demand for money- for the period 1850-1997. A cointegration analysis and an error correction model investigate the importance of the institutional variables in explaining the long-run behavior of the income velocity of money in Spain. The findings reveal a greater stability in the long-run velocity model when the institutional variables are considered.
\end{abstract}

Keywords: velocity of circulation, money demand, Spain, 1850-2000 JEL Classification: E41, N10, N13, N14

\title{
1. INTRODUCCIÓN
}

Diversos estudios han estimado e intentado explicar el comportamiento de largo plazo de la velocidad de circulación del dinero (V, en adelante) en una serie de países industrializados (Bordo y Jonung, 1981, 1987, 2004; Saint-Marc, 1983; Fratianni y Spinelli, 1991; Siklos, 1993; Bordo et al., 1997). Variables tan importantes como la monetización, la sofisticación financiera y la construcción del estado de bienestar contribuyen de manera muy significativa a la explicación de su patrón de comportamiento. De manera muy general, podemos decir que son cambios en las instituciones monetarias y financieras la base fundamental de los cambios de tendencia de la V. De acuerdo con Bordo y Jonung (1981), la 
V ha seguido, a lo largo del siglo largo posterior a 1870, una forma de «U» muy abierta. Según ellos, la tendencia descendente de la V queda explicada a través de los procesos de monetización mientras que la ascendente se debe al aumento de la sofisticación del sistema financiero, así como al aumento de la estabilidad y seguridad económicas reflejados en el desarrollo del estado de bienestar. Sin embargo, en España, un análisis de este calibre no ha sido nunca llevado a cabo para la V fundamentalmente por la falta de las series temporales necesarias.

Gracias al esfuerzo realizado por una generación de investigadores económicos españoles, hoy en día disponemos de series temporales suficientemente largas y fiables que nos permiten profundizar en el desarrollo y comportamiento a largo plazo de la V en España, así como proponer interpretaciones de su evolución.

El interés de intentar la cuantificación de la V para España entendemos que es cuádruple. En primer lugar, estimar una variable fundamental, existente para todos los países desarrollados y de la que carecemos en España. Es un reto que hay que superar y que nos debiera permitir abrir nuevas perspectivas problemáticas y de investigación, amén de facilitarnos un encaje comparativo internacional del caso español. En segundo lugar, la V nos conduce a preguntarnos por sus determinantes, perfilarlos y estimar si son realmente explicativos. En tercer lugar, la reflexión sobre la $\mathrm{V}$ nos conduce inmediatamente a la demanda de dinero, su inverso. En cuarto lugar, la V nos debiera iluminar la historia monetaria española y proporcionarnos claves más depuradas para definir sus etapas y sus rasgos característicos. En trabajos precedentes se había supuesto su estabilidad o la regularidad de su pauta temporal, mientras que a nosotros nos interesa su gran variabilidad. Pese al interés de esta temática, las limitaciones de espacio nos impedirán tratarla en esta ocasión.

En lo que sigue comenzaremos con la presentación de la estimación de la V. Consideraremos, a continuación, la V en el contexto internacional y los rasgos distintivos de su evolución a largo plazo en España. La cuarta parte —y la más larga - la dedicaremos al análisis de los determinantes de la V: cuáles podrían ser, cómo estimarlos y cómo encajarlos en un modelo explicativo. Ello nos llevará, de hecho, a interesarnos por la función de demanda de dinero y su estimación. En las conclusiones reflexionaremos sobre los resultados obtenidos.

\section{ESTIMACIÓN}

La definición usual de la $\mathrm{V}$ deriva de la tradicional fórmula de la teoría cuantitativa del dinero: $\mathrm{MV}=\mathrm{PT}$, siendo $\mathrm{M}$ la oferta monetaria; $\mathrm{V}$, la velocidad de circulación del dinero; P, el nivel de precios, y T, el volumen 
de transacciones. A lo largo de las últimas décadas, comenzando con la revisión monumental que realizaron Friedman y Schwartz (1963) de la historia monetaria de los Estados Unidos, y siguiendo con la síntesis comparativa de Bordo y Jonung (1987), se ha venido usando M2 como la magnitud más representativa de la oferta monetaria, el deflactor del PIB como la más representativa del nivel de precios y la más pertinente en este caso y el PIB a precios de mercado como la mejor aproximación a la T.

Definimos la oferta monetaria (M2) como efectivo en manos del público, depósitos a la vista, depósitos de ahorro y depósitos a plazo - disponibilidades líquidas (una M3 restringida o una M2 amplia)—. Los trabajos de Martín Aceña (1985a, 1985b, 1989, 2000 y, junto a Pons, 2005) han ido definiendo, refinando y ampliando las series de M2. La que aquí usamos se basa en los cálculos de Martín Aceña (2000) para el período 18481873, y Martín Aceña (1989) para el período 1874-1980. Los últimos años proceden del Boletín Estadístico del Banco de España (2001), pero la actualización más allá de 1997 es espuria, pues no corresponde, propiamente, al mismo fenómeno que la anterior, tal como Martín Aceña y Pons (2005) subrayan. En efecto, el Banco de España no proporciona series comparables de oferta monetaria a partir de 1998 a causa de la creación del euro y del Banco Central Europeo. La serie de oferta monetaria no se encuentra disponible durante los años de 1936 a 1940.

Las viejas series de precios al por mayor (Maluquer de Motes, 1989) han sido enriquecidas por un conjunto bien trabado de series de precios al consumo y al por mayor (Maluquer de Motes, 2005), más completos en su cobertura territorial, en su cesta de productos y en el rigor de su elaboración, que han culminado en la estimación de un deflactor del PIB a precios de mercado de amplia cobertura (Prados de la Escosura, 2003).

Los datos del PIB, que en estimaciones anteriores de la V eran la incógnita que se deseaba despejar, ahora son una base sólida para nuestras investigaciones. La serie de Producto Interior Bruto (PIB) en términos nominales y a precios de mercado, de 1850 a 2000, de Prados de la Escosura (2003, cuadro A.13.2), ampliamente utilizada por todos los investigadores, es un ingrediente fundamental en nuestra estimación. Con todos estos mimbres vamos a tratar de componer un nuevo cesto: la $\mathrm{V}$ española.

Nuestras principales diferencias con las estimaciones precedentes (Tortella, 1983, 1994 y 1998; Coll, 1989 y 1992; Martín Aceña, 1990 —ver el Apéndice A para su consideración más detallada-) consisten en haber sustituido algunas de las piezas estadísticas (PIB y precios) y haber alargado el ejercicio en las dos direcciones: de 1900 hacia atrás hasta 1850 y de 1980 en adelante hasta el año 2000. Combinados, todos los cambios que hemos introducido proporcionan un nuevo estimador de la V que consideramos que es sólido, todo lo sólido que puede ser en términos internacionales y en términos históricos. 
En lo que sigue usamos siempre la V2 —la velocidad de circulación del dinero calculada con la M2 - por ser aquella sobre la que se han realizado todas las comparaciones internacionales. El cuadro 1 y el gráfico 1 presentan la serie V2 del año 1850 al 2000.

CUADRO 1

VELOCIDAD DE CIRCULACIÓN DEL DINERO (V2), ESPAÑA, 1850-2000

\begin{tabular}{|c|c|c|c|c|c|c|c|}
\hline Año & V2 & Año & V2 & Año & V2 & Año & V2 \\
\hline 1850 & 7,075 & 1888 & 3,997 & 1926 & 2,780 & 1964 & 1,506 \\
\hline 1851 & 7,121 & 1889 & 3,566 & 1927 & 2,804 & 1965 & 1,489 \\
\hline 1852 & 7,078 & 1890 & 3,590 & 1928 & 2,567 & 1966 & 1,504 \\
\hline 1853 & 8,221 & 1891 & 3,608 & 1929 & 2,580 & 1967 & 1,459 \\
\hline 1854 & 8,098 & 1892 & 3,714 & 1930 & 2,425 & 1968 & 1,365 \\
\hline 1855 & 8,045 & 1893 & 3,683 & 1931 & 2,442 & 1969 & 1,306 \\
\hline 1856 & 6,827 & 1894 & 3,888 & 1932 & 2,422 & 1970 & 1,261 \\
\hline 1857 & 6,223 & 1895 & 3,663 & 1933 & 2,262 & 1971 & 1,151 \\
\hline 1858 & 5,808 & 1896 & 3,400 & 1934 & 2,418 & 1972 & 1,101 \\
\hline 1859 & 5,648 & 1897 & 3,393 & 1935 & 2,407 & 1973 & 1,079 \\
\hline 1860 & 5,622 & 1898 & 2,705 & 1936 & $\ldots$ & 1974 & 1,123 \\
\hline 1861 & 5,357 & 1899 & 2,777 & 1937 & $\ldots$ & 1975 & 1,123 \\
\hline 1862 & 5,089 & 1900 & 3,043 & 1938 & $\ldots$ & 1976 & 1,115 \\
\hline 1863 & 5,145 & 1901 & 3,132 & 1939 & $\ldots$ & 1977 & 1,190 \\
\hline 1864 & 4,709 & 1902 & 3,185 & 1940 & $\ldots$ & 1978 & 1,219 \\
\hline 1865 & 4,256 & 1903 & 3,279 & 1941 & 1,616 & 1979 & 1,204 \\
\hline 1866 & 4,472 & 1904 & 3,481 & 1942 & 1,808 & 1980 & 1,211 \\
\hline 1867 & 4,456 & 1905 & 3,479 & 1943 & 1,817 & 1981 & 1,171 \\
\hline 1868 & 3,679 & 1906 & 3,571 & 1944 & 1,836 & 1982 & 1,189 \\
\hline 1869 & 3,269 & 1907 & 3,713 & 1945 & 1,623 & 1983 & 1,202 \\
\hline 1870 & 3,400 & 1908 & 3,749 & 1946 & 1,668 & 1984 & 1,217 \\
\hline 1871 & 3,638 & 1909 & 3,656 & 1947 & 1,675 & 1985 & 1,274 \\
\hline 1872 & 4,148 & 1910 & 3,410 & 1948 & 1,688 & 1986 & 1,405 \\
\hline 1873 & 4,428 & 1911 & 3,572 & 1949 & 1,639 & 1987 & 1,466 \\
\hline 1874 & 4,399 & 1912 & 3,478 & 1950 & 1,761 & 1988 & 1,483 \\
\hline 1875 & 4,260 & 1913 & 3,603 & 1951 & 1,909 & 1989 & 1,506 \\
\hline 1876 & 4,207 & 1914 & 3,523 & 1952 & 1,833 & 1990 & 1,385 \\
\hline 1877 & 4,671 & 1915 & 3,496 & 1953 & 1,786 & 1991 & 1,347 \\
\hline 1878 & 4,471 & 1916 & 3,565 & 1954 & 1,777 & 1992 & 1,400 \\
\hline 1879 & 4,547 & 1917 & 3,182 & 1955 & 1,649 & 1993 & 1,324 \\
\hline 1880 & 4,558 & 1918 & 3,011 & 1956 & 1,743 & 1994 & 1,322 \\
\hline 1881 & 4,579 & 1919 & 2,851 & 1957 & 1,774 & 1995 & 1,339 \\
\hline 1882 & 4,816 & 1920 & 2,962 & 1958 & 1,839 & 1996 & 1,381 \\
\hline 1883 & 4,809 & 1921 & 2,750 & 1959 & 1,806 & 1997 & 1,440 \\
\hline 1884 & 4,583 & 1922 & 2,516 & 1960 & 1,619 & 1998 & 1,422 \\
\hline 1885 & 4,292 & 1923 & 2,420 & 1961 & 1,594 & 1999 & 1,375 \\
\hline 1886 & 4,373 & 1924 & 2,682 & 1962 & 1,544 & 2000 & 1,383 \\
\hline 1887 & 3,993 & 1925 & 2,821 & 1963 & 1,595 & & \\
\hline
\end{tabular}

Fuente: Ver texto. 


\section{GRÁFICO 1}

VELOCIDAD DE CIRCULACIÓN DEL DINERO (V2) EN ESPAÑA, 1850-2000

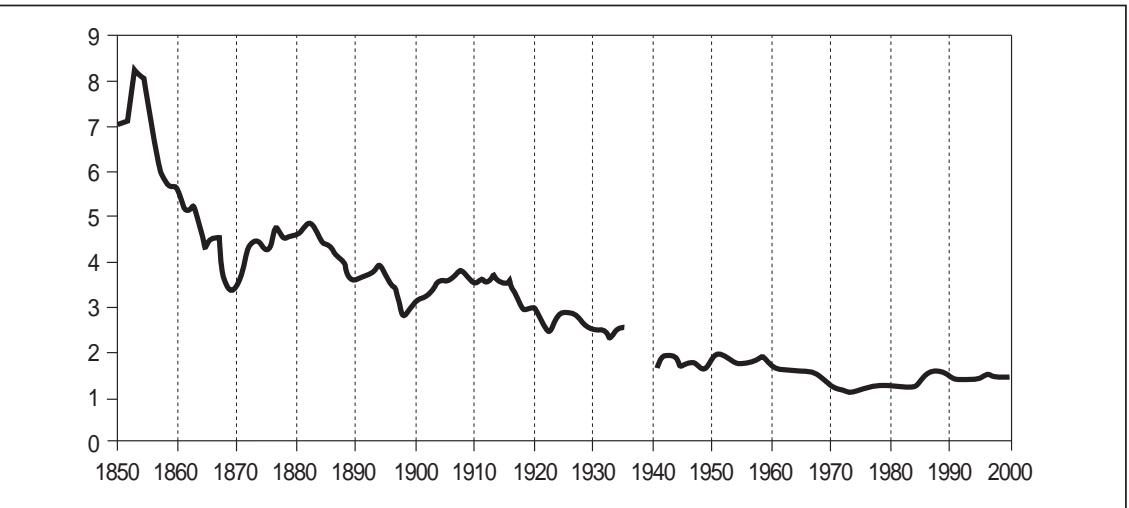

Fuentes: Elaboración propia. Ver texto.

\section{LA COMPARACIÓN INTERNACIONAL EN EL LARGO PLAZO}

Durante los últimos veinte años, dos historiadores económicos - Michael Bordo y Lars Jonung — han estudiado sistemáticamente la V para numerosos países. Basándose en su propia investigación de base y movilizando la de otros, han reconstruido las tendencias más estilizadas de la V y sus determinantes. En una síntesis telegráfica, Bordo y Jonung plantean que la $\mathrm{V}$ ha seguido, a lo largo del siglo largo posterior a 1870, una forma de «U» muy abierta. En casi todos los países, la V era alta hacia 1870 y ha disminuido con bastante regularidad desde entonces hasta 1913. En algunos casos la disminución ha continuado durante el período de entreguerras. Normalmente, en algún momento posterior a la segunda guerra mundial (pero hay casos en que el fenómeno se da antes), la caída llega a un mínimo y la $\mathrm{V}$ vuelve a aumentar. Este rasgo parece bien establecido para la V2 (la velocidad que usa la M2). El «rebote» de la serie prosigue, apaciblemente, hasta el final de su estudio ${ }^{1}$. El nivel mínimo suele estar en torno al valor uno (la oferta monetaria equivale al PIB monetario).

Bordo y Jonung interpretan que la larga caída de la V se debe no sólo a la creciente monetización de la economía sino también al desarrollo del sistema de bancos comerciales. A fin de cuentas, ambos son componentes del proceso de modernización económica. Los países con moder-

1 El decenio de 1980 en su primera versión (1987), y el año 2000 en la segunda (2004). 
nizaciones precoces, como Gran Bretaña y Francia, son algo diversos, pues han experimentado antes, o a lo largo de un proceso mucho más dilatado en el tiempo, el mismo fenómeno (Capie, 2004). Los países de desarrollo tardío deberían haber experimentado la caída secular de la V con retraso. En un orden de cosas algo distinto, Bordo y Jonung observan (siguiendo a Friedman y Schwartz) que cuanto más amplia es la definición de dinero, más se aprecia esta pauta de «U» abierta ${ }^{2}$. El proceso de monetización llega a su fin y con él la caída de la $V$ por dos motivos fundamentales. En primer lugar, cuando aparecen los primeros sustitutos del dinero como forma de pago - lo que se viene a denominar sofisticación financiera (por ej., las tarjetas de crédito) - y segundo, cuando la extensión del estado del bienestar reduce de la misma manera la demanda de dinero por motivo de precaución. Los ciudadanos dejan de ahorrar si el ahorro ya se produce mediante sus cotizaciones a la seguridad social o mediante sus impuestos. Cuanto más precoz y más general es la implantación de la red de seguridad social, antes se interrumpe la caída de la V. En los países más atrasados, deberemos observar un cambio de tendencia más tardío. Con los datos que han conseguido movilizar, Bordo y Jonung presentan evidencia convincente de la existencia de todos estos fenómenos. En todos los casos, ellos se limitan al análisis de la V derivada de la M2 y lo mismo haremos nosotros.

España encaja bien en este esquema. Hemos planteado una comparación esquemática de la experiencia española y de la de los demás países cubiertos por la encuesta de Bordo y Jonung en los cuadros siguientes. Comenzamos por fijarnos en los niveles máximos alcanzados por la V (cuadro 2). El cuadro está clasificado en orden decreciente de los valores máximos registrados. El máximo español, de 1853, es de 8,2, por debajo del valor alcanzado por Dinamarca en 1871, y por encima del de Suecia en el mismo año, y de los de Canadá en 1876 e Italia en 1861. En general, los máximos corresponden a alguno de los primeros años de las series disponibles. Así es para Dinamarca, España, Suecia, Canadá, Italia, Estados Unidos y Noruega. La experiencia española no desentona ni en los niveles ni en la cronología. De quien sí queda más lejos es de los países con monetizaciones precoces, como Gran Bretaña, Francia o Alemania, y de aquellos que, por su misma precocidad, no tienen el registro de $\mathrm{V}$ más alto a mediados o finales del siglo XIX sino a finales del siglo Xx (Australia y Gran Bretaña).

Los máximos de las series son interesantes en sí mismos. ¿Existe un máximo «natural»? El valor más alto registrado en el cuadro 2 es 9,4 para Dinamarca en 1871. ¿Qué valores pudieron darse para períodos

2 Con definiciones más restrictivas de dinero (M0 o M1), no hay caída de la V, sino que puede suceder lo contrario, que haya subida secular o estancamiento. 


\section{CUADRO 2}

COMPARACIÓN INTERNACIONAL DE LOS NIVELES MÁXIMOS ALCANZADOS POR LA VELOCIDAD DE CIRCULACIÓN DEL DINERO (V2), 1870-1986*

\begin{tabular}{|c|c|c|}
\hline & Valor Máximo & Año \\
\hline Dinamarca.... & 9,4 & 1871 \\
\hline 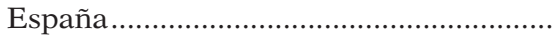 & 8,2 & 1853 \\
\hline Suecia & 6,7 & 1871 \\
\hline 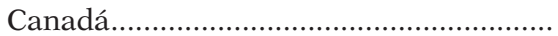 & 6,5 & 1876 \\
\hline 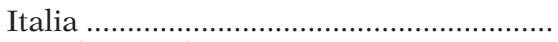 & 6,2 & 1861 \\
\hline 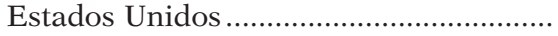 & 5,7 & 1869 \\
\hline Holanda & 5,6 & 1903 \\
\hline Japón & 4,0 & 1891 \\
\hline 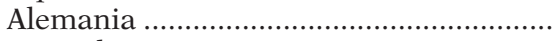 & 4,0 & 1925 у 1951 \\
\hline Australia & 4,0 & 1970 \\
\hline Francia & 3,6 & $1855-1856$ \\
\hline Noruega & 3,6 & 1873 \\
\hline Finlandia & 3,2 & 1951 \\
\hline Gran Bretaña .............................................. & 2,4 & 1971 \\
\hline
\end{tabular}

Fuentes: Para España, ver texto. Francia: Saint-Marc (1983). Italia: Spinelli \& Fratianni (1991). Estados Unidos y Gran Bretaña: Friedman y Schwartz (1982). Para el resto, Bordo y Jonung (1981, 1987) y Siklos (1993).

Nota: * Para España y Francia consideramos la serie desde 1850, para Italia desde 1861 y para Estados Unidos, 1869.

anteriores? La única respuesta nos la pueden dar las historias monetarias nacionales. En la medida de lo posible, hemos reunido información sobre todos aquellos países con investigación publicada al respecto. Hemos observado estimaciones de la $\mathrm{V}$ en el rango de 10-11 para los Estados Unidos en los primeros años del decenio de 1820 (Temin, 1969). Para Inglaterra, el máximo registrado en la literatura es de 9,3 y corresponde a 1561 (Mayhew, 1995). Hay indicios suficientes para sugerir que los niveles españoles - muy altos a principios del decenio de 1850también podrían haberse situado en torno a 10 para los decenios de 1830 y 1840 (Sudriá y Pascual, 1999; Tedde, 1999; García López, 2001). Los altos valores estadounidenses están relacionados con un período de aguda escasez de bancos comerciales que se solucionaría en los años siguientes. Lo mismo podría haber sucedido en España, donde los primeros bancos comerciales constituidos como sociedades anónimas aparecen en 1844 (Sánchez-Albornoz, 1975). Es probable que los años inmediatamente precedentes fueran un máximo histórico en la velocidad de circulación del dinero.

Los puntos de inflexión (mínimos seguidos por cambios de tendencia permanentes) suelen ser más precoces en todos los demás países (véase 


\section{CUADRO 3 \\ COMPARACIÓN INTERNACIONAL DE LOS PUNTOS \\ DE INFLEXIÓN EN LAS SERIES DE LA VELOCIDAD DE CIRCULACIÓN DEL DINERO (V2), 1870-1986}

\begin{tabular}{|c|c|c|}
\hline & Año & Valor de inflexión \\
\hline Alemania ...... & 1912 & 1,2 \\
\hline  & 1913 & 1,4 \\
\hline & 1934 & 1,5 \\
\hline Suecia. & 1922 & 1,5 \\
\hline Noruega... & 1922 & 0,9 \\
\hline & 1945 & 0,8 \\
\hline 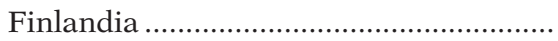 & 1940 & 1,4 \\
\hline Italia ............ & 1943 & 0,9 \\
\hline 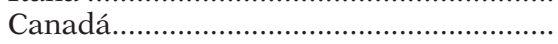 & 1945 & 1,7 \\
\hline 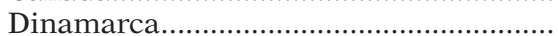 & 1945 & 1,9 \\
\hline 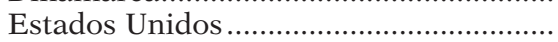 & 1946 & 1,2 \\
\hline Australia & 1946 & 1,7 \\
\hline 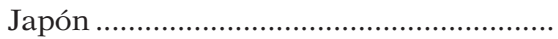 & 1946 & 1,7 \\
\hline 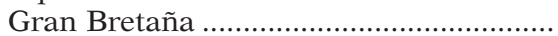 & 1,1 & \\
\hline Holanda & 1948 & 1,8 \\
\hline España & 1973 & 1,1 \\
\hline
\end{tabular}

Fuentes: Para España, ver texto. Francia: Saint-Marc (1983). Italia: Spinelli \& Fratianni (1991). Estados Unidos y Gran Bretaña: Friedman y Schwartz (1982). Para el resto, Bordo y Jonung (1981, 1987, 2004) y Siklos (1993).

el cuadro 3). Algunos lo exhiben para 1912 (Alemania, pero faltan muchos datos en su serie) ó 1913 (Francia, con otro valor muy similar para 1934), o para 1922 (Suecia y Noruega, con otro punto alternativo en 1945). También los hay para inmediatamente antes de la Segunda Guerra Mundial: 1939/40 (Alemania y Finlandia). Es más frecuente encontrarlo en la inmediata segunda posguerra mundial: 1943, Italia; 1945, Canadá y Dinamarca; 1946, Australia, Estados Unidos y Japón; 1947, Gran Bretaña, y 1948, Holanda. Desgraciadamente, numerosos países sufren de falta de datos para algunos momentos particularmente tempestuosos en su historia monetaria y ello podría alterar algo la cronología que proponemos. España es excepcional por lo retrasado de su punto de inflexión: 1973. Si las razones del cambio de tendencia consistieran, fundamentalmente, en el despliegue del estado del bienestar, el caso español quedaría muy bien enmarcado. En efecto, sólo es a finales de la época franquista que comienzan a generalizarse los elementos constitutivos del estado del bienestar en España: cobertura médica de la seguridad social, educación obligatoria gratuita, pensiones de jubilación, etc. (Comín, 1996). En una década, la que va de los últimos años de esplendor económico del tardofranquismo, al primer gobierno socia- 
lista, pasando por la transición y los gobiernos de la UCD, se plantea y despliega el estado del bienestar en España. Ahora bien, si el elemento dominante en la explicación es la completa monetización derivante de las experiencias de fiduciarización extrema vinculadas a las excepcionales coyunturas bélicas (que probablemente se acerca más a la realidad histórica) y al abandono del patrón oro, entonces deberemos introducir dos consideraciones para España. La primera es que nos faltan datos para los años de la Guerra Civil. Pero podemos deducir de la investigación histórica disponible que la oferta monetaria en la zona franquista estuvo bajo control y que el descontrol emisor de la zona republicana estuvo presidido por la resistencia de la autoridad monetaria a multiplicar la emisión de moneda y billetes, lo que conllevaba una tremenda escasez de circulante (Martín Aceña, 1985b; Velarde, 2000; Martorell, 2005). La segunda consideración es que el caso español sólo es plenamente comparable al de los países que no experimentaron ninguna gran guerra - los neutrales de las dos conflagraciones internacionales del siglo $\mathrm{xx}$ - o que no la sufrieron en su propio territorio. Ahí la comparación más pertinente es con Suecia, lo que nos permite volver a conceder verosimilitud al motivo precaución. La comparación con los Estados Unidos también es tranquilizante.

Más allá de la esquematización que representa comparar máximos y puntos de inflexión, interesa comparar la trayectoria de la serie española con la de otros países relevantes. Hemos considerado cuatro: Estados Unidos, Reino Unido, Francia e Italia. Los dos primeros constituyen una referencia indispensable, tanto por su liderazgo económico en el período considerado, como por ser los más y mejor estudiados en obras ya clásicas (Friedman y Schwartz, 1982). Los dos últimos son vecinos importantes y disponen de monografías exhaustivas sobre su historia monetaria y de estimaciones aceptadas sobre la evolución de la V. En el gráfico 2 presentamos la evolución del caso español comparada con la del estadounidense y la del británico. Nos hemos debido limitar al período 1869-1975 para adecuarnos a la cobertura de las series estimadas por Friedman y Schwartz.

Si el nivel de la $\mathrm{V}$ es indicativo del grado de monetización, no hay duda del retraso español respecto al Reino Unido. Sólo después de la Guerra Civil pasó España a alcanzar niveles comparables a los de los países occidentales más avanzados. Sin embargo, la comparación con Estados Unidos pone de manifiesto que, a la altura del decenio de 1870 la V de ambos países era muy similar. Después de 1880 la estadounidense siguió bajando con decisión mientras que la española tendió a estancarse. Los episodios de reducción de la V fueron transitorios (como sucedió a finales del siglo XIX) o se retrasaron significativamente. Sólo tras la Guerra Civil convergen los niveles. 


\section{GRÁFICO 2}

LA VELOCIDAD DE CIRCULACIÓN DEL DINERO (V2)

EN ESPAÑA, ESTADOS UNIDOS Y EL REINO UNIDO, 1869-1975

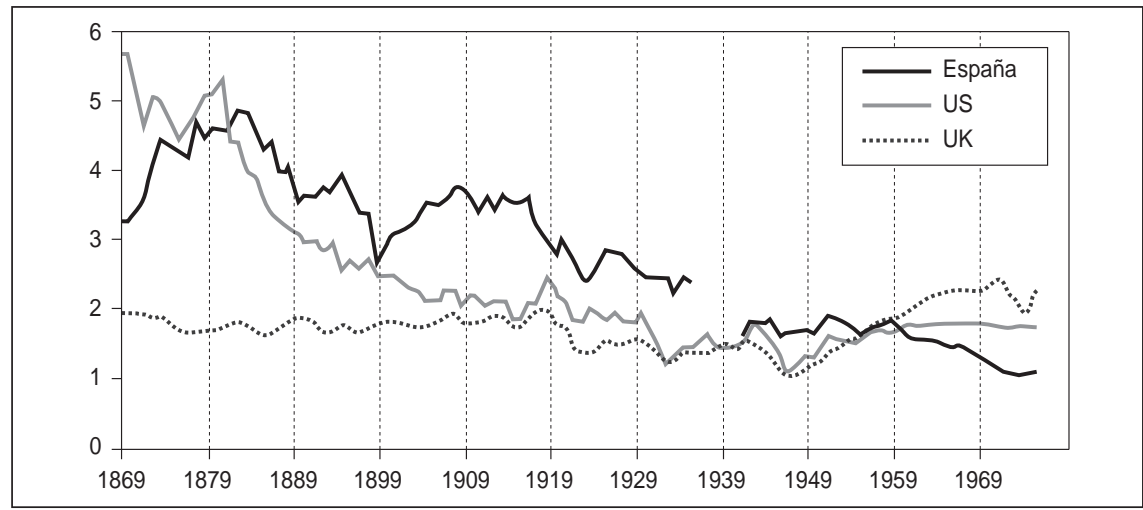

Fuentes: Estados Unidos y Reino Unido, Friedman y Schwartz (1982, cuadros 4.8 y 4.9). España, cuadro 1.

La experiencia española se compara bastante bien con la italiana (véase el gráfico 3). Los niveles y tendencias son casi idénticos en el decenio de 1860, cuando ambos países experimentan reducciones de su V, de similar nivel e intensidad. Pero, mientras que Italia continúa con la caída

GRÁFICO 3

LA VELOCIDAD DE CIRCULACIÓN DEL DINERO (V2)

EN ESPAÑA, FRANCIA E ITALIA, 1850- 1980

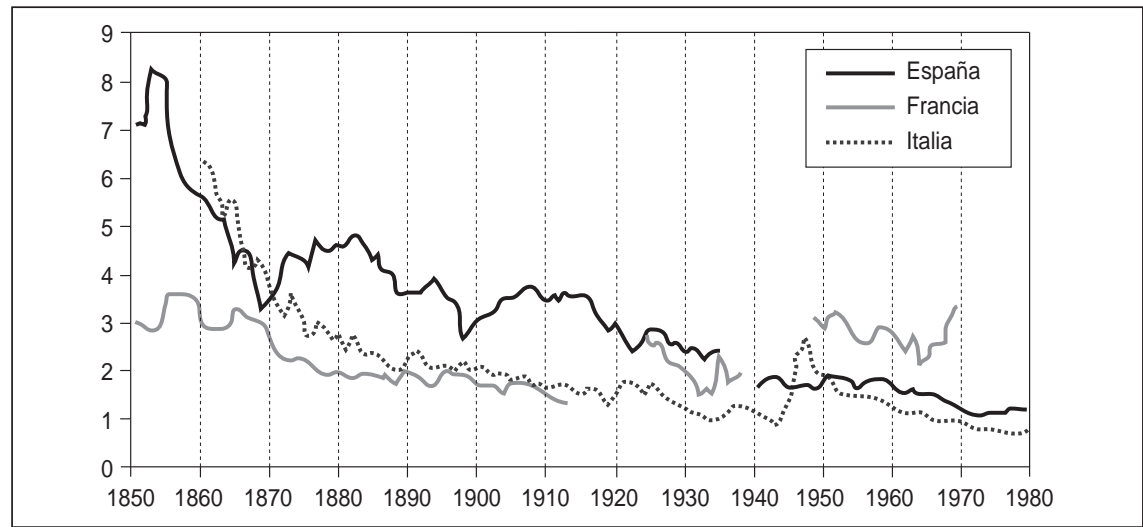

Fuentes: Francia, Saint-Marc (1983); Italia: Spinelli \& Fratianni (1991); España, cuadro 1. 
de la V, en modo parecido a los Estados Unidos, España interrumpe el proceso durante cuatro decenios. De 1910 en adelante la V española sigue el curso de la italiana, pero a niveles netamente superiores. Sólo convergirán después de la Segunda Guerra Mundial En comparación con Francia, España parte de un nivel mucho más alto, lo que no debe sorprendernos: Francia era mucho más próspera que España y resultaba natural que su demanda de dinero fuera también superior. La tendencia española es a la convergencia, pero con lentitud. Sólo se produce en el período de entreguerras, cuado coincide una caída de la demanda de dinero en Francia y una subida en España. El resultado es una fuerte coincidencia de los niveles y tendencias de las $\mathrm{V}$ de ambos países.

En la comparación con Estados Unidos e Italia, y manteniendo el rabillo del ojo atento a lo que nos puede enseñar el caso sueco (Bordo \& Jonung, 2004), quizá lo más destacable de la trayectoria española es la lentitud de la caída de la V desde 1870 a 1914. En rigor, no hay caída, pues se pasa de 3,4 a 3,5. Es verdad que 1870 es un punto anormalmente bajo. Tres años antes o tres años después estaba en torno a 4,4. Pues bien, ese modestísimo punto de reducción de la V contrasta con las caídas de tres y cuatro puntos habituales en Estados Unidos, Canadá, Suecia y Dinamarca (en este último caso, siete puntos). También contrasta con las caídas más modestas — del orden de dos puntos o dos puntos y medio- pero muy continuas, sin interrupciones significativas, de Noruega, Alemania, Italia y Japón (en este caso, desde 1890). Claro está que más estables son las trayectorias británica, francesa y australiana. Como en otras ocasiones, la época 1870-1914 sugiere una trayectoria española más próxima a la de las economías maduras de la Europa occidental que a la de las economías periféricas europeas o ultramarinas, abocadas abiertamente, y con mucho éxito, a largas etapas de crecimiento rápido. En otras palabras, la trayectoria de la V parece inversamente proporcional al PIB. No nos debe extrañar. Otra forma de argumentar lo mismo es enunciar que la demanda de dinero es directamente proporcional al PIB. En un mundo básicamente vinculado a patrones metálicos de pleno contenido, no podía ser de otra manera.

La gran caída de 1855-1869 podría ser la que echamos en falta después de 1870. Como en tantos otros elementos de la economía española de la segunda mitad del siglo Xx, las grandes reformas del decenio de 1850, que aspiraban a una fuerte modernización económica, parecen haberse diluido en el tiempo sin dar lugar a un cambio económico lo suficientemente potente como para generar un proceso de convergencia como el que se produce en Alemania, en los países escandinavos o en Italia.

$\mathrm{Si}$, en cambio, miramos la $\mathrm{V}$ española en logaritmos en lugar de en valores absolutos (véase el gráfico 4), apreciaremos más la continuidad 


\section{GRÁFICO 4}

VELOCIDAD DE CIRCULACIÓN DEL DINERO (V2),

EN LOGARITMOS. ESPAÑA, 1850-2000

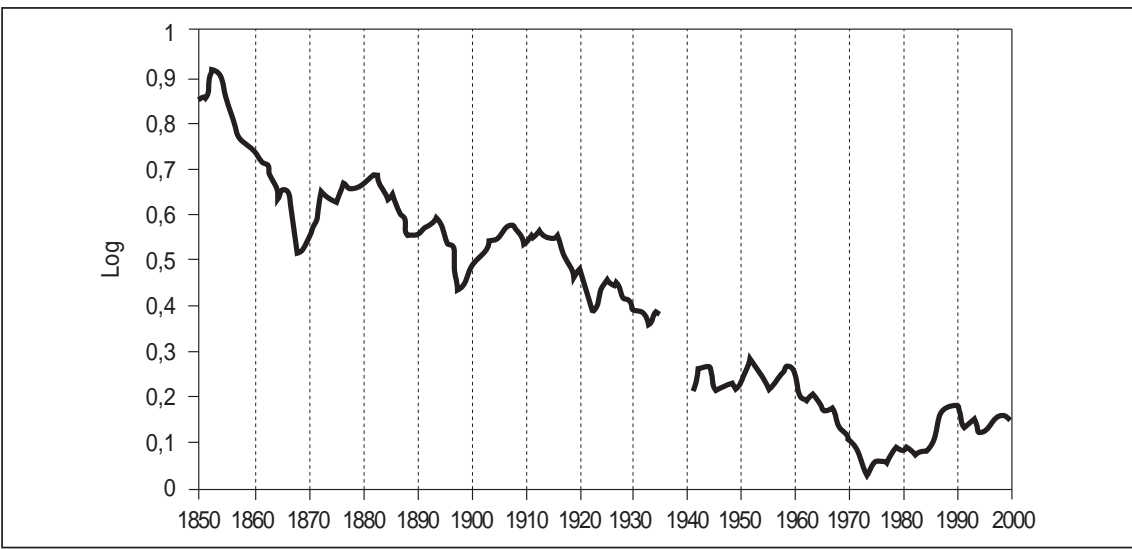

Fuentes: Elaboración propia. Ver texto.

en las tasas de reducción de la V y la neta aceleración de su caída que se produce entre 1951 y 1973. Ésta sólo sería comparable a la de 1855-1869. En términos internacionales no tenemos ejemplos bien documentados de este período, pero sí de aquél. En efecto, tanto Italia como Japón experimentaron caídas dramáticas y prolongadas de su V en la segunda posguerra mundial.

Otro rasgo de la serie española que quisiéramos subrayar es su carencia de cambios radicales o de comportamientos erráticos. En esto, de nuevo, se parece más a los países norteamericanos y escandinavos. La neutralidad en las grandes guerras o el no sufrirlas en su propio territorio podría explicar parte de ello, aunque no parece que todo. Hay ocasiones que se asemejan a cambios de régimen, como las bruscas inflexiones de 1869 y de 1898-1899.

\section{ANÁLISIS EMPÍRICO}

La visión tradicional sobre la velocidad de circulación del dinero explica su comportamiento a través de dos variables fundamentales como son el coste de oportunidad del dinero, así como la renta real, o la renta real permanente. Aparte de esta visión tradicional existe la visión institucional sobre el comportamiento secular de la velocidad del dinero que suma a esas variables tradicionales las variables institucionales que 
pueden añadir poder explicativo al comportamiento de la serie (Bordo y Jonung, 1987). Alternativamente la velocidad de circulación debe de pensarse como un paseo aleatorio, es decir una serie temporal que en el largo plazo no es estacionaria o lo que es lo mismo tiene una raíz unitaria. Sin embargo, el hecho de que la velocidad de circulación tenga una raíz unitaria no implica que una combinación lineal de las variables que la definen no pueda tener una tendencia en el largo plazo hacia un determinado valor. Por consiguiente, un análisis econométrico que definiera la naturaleza de una relación a largo plazo entre las variables tradicionales y las variables institucionales determinantes de la $\mathrm{V}$ española permitiría determinar hasta qué punto los cambios institucionales explican el comportamiento de la velocidad.

El objetivo de este apartado es presentar y analizar de forma empírica la velocidad de circulación del dinero en España en el muy largo plazo para poder dar una explicación al comportamiento secular de la serie usando no sólo las variables tradicionales como son la renta y el tipo de interés sino también una serie de variables institucionales que enfatizan la influencia en la velocidad del proceso de monetización, el crecimiento de los bancos comerciales y el desarrollo del sistema financiero. Entonces y para poder medir los efectos institucionales en la velocidad del dinero debemos construir unas series que nos permitan medir los efectos de la monetización y de la sofisticación del sistema financiero usando unas variables proxies que se ajusten a los datos españoles de los que disponemos.

En primer lugar y para calcular la monetización usamos como proxy la serie de la composición del empleo que explica como según la economía se va desarrollando y creciendo existe un desplazamiento desde la agricultura a la industria, el comercio y los servicios. Se trata de medir la proporción de población activa no agraria. En segundo lugar y para medir la sofisticación del sistema financiero creamos una nueva variable calculada como la relación entre el dinero legal y las disponibilidades líquidas que refleja como al crecer la economía, su estructura financiera va cambiando y se va desarrollando siendo esta nueva variable un buen proxy del desarrollo financiero.

De esta forma, estas dos variables institucionales definen conjuntamente el comportamiento de la velocidad de circulación. En primer lugar se observa el efecto de la monetización que produce la caída de la velocidad mientras que, por el contrario, el efecto de la sofisticación financiera se observa a partir del punto de inflexión cuando la velocidad empieza una trayectoria ascendente. La fuerza relativa de estas dos variables determina el momento exacto de la inflexión de la velocidad de circulación explicando la forma de U característica de esta serie (véase el gráfico 4). 
El análisis básico para estudiar la relación de la variables tradicionales e institucionales con la velocidad del dinero es una estimación de la función de la velocidad derivada de la ecuación tradicional de la demanda de dinero medida en logaritmos naturales (excepto para el tipo de interés).

$$
\begin{gathered}
\log \mathrm{V}=\mathrm{A}_{0}+\left(1-\mathrm{A}_{1}\right) \log \mathrm{Y}^{\mathrm{p}}+\mathrm{A}_{2} i+\mathrm{A}_{3} \log \text { ciclo }+\mathrm{A}_{4} \log \text { nalf }+ \\
+\mathrm{A}_{5} \log \text { sof }+\mathrm{A}_{6} \log \text { def }+\mathrm{e}
\end{gathered}
$$

donde $\mathrm{Y}^{\mathrm{p}}$ representa la renta permanente real per cápita; $i$ es el tipo de interés que representa el coste de oportunidad de tener dinero; ciclo es la relación entre la renta real y la renta real permanente per cápita; nalf representa la composición del empleo y por tanto el proceso de monetización; sof es la medida de la sofisticación del sistema financiero; y por último la variable def es la diferenciación de la serie del deflactor del PIB nominal que utilizamos como medida de la inflación. Hay que notar que los coeficientes $\mathrm{A}_{1}$ y $\mathrm{A}_{2}$ representan la elasticidad de la renta permanente y la semi-elasticidad del tipo de interés, respectivamente, para la demanda de dinero. De igual forma los otros coeficientes representan la elasticidad para la velocidad de las variables correspondientes.

Para analizar con detalle el significado de estas variables empezaremos por las variables tradicionales que determinan la función de la demanda de dinero, a saber, la renta real permanente per cápita y el tipo de interés. La primera variable se ha calculado siguiendo la hipótesis de la renta real permanente de Friedman (1957) y sus ponderadores junto con el crecimiento de la renta real per cápita en el largo plazo (ver el apéndice B). La segunda variable, el tipo de interés, es una combinación de las diversas series de interés en el largo plazo disponibles en España (ver el apéndice B) ${ }^{3}$.

A parte de estas dos variables fundamentales, nos hemos visto obligados a incluir una variable más que pueda medir el efecto que la inflación tuvo sobre la velocidad de circulación. Después de la Guerra Civil se inaugura un largo período caracterizado por tasas de inflación significativas, bien distinto al anterior a 1936. Estas altas tasas de inflación afectan a la demanda de dinero. Además el hecho de que en España y durante los años posteriores a la Guerra Civil hubiera un control gubernativo del mercado monetario y de capitales, nos obliga aún más a considerar esta variable como fundamental en la definición de la función de velocidad ya que el tipo de interés deja de ser una medida fiable del coste de oportunidad de tener dinero (Bordo y Jonung, 1981). Por eso y en estas circunstancias consideramos que una medida del cambio de pre-

\footnotetext{
3 Véase Bordo y Jonung, 1981.
} 
cios basada en la serie del deflactor del PIB diferenciada, def (ver el apéndice B), puede ser necesaria a la hora de definir el comportamiento de la velocidad de circulación.

Las variables institucionales son medidas a través de proxies de las que hemos hablado con anterioridad y por tanto sólo nos quedaría indicar la relación que tienen con la velocidad. La medida de la sofisticación financiera está positivamente correlacionada con la velocidad - explica su trayectoria ascendente- - La monetización, por el contrario, presenta una relación inversa con la velocidad y está positivamente correlacionada con la demanda de dinero - explica la trayectoria descendente de la V-.

Por último, la variable ciclo que hemos incluido en la regresión pretende capturar el efecto de la renta no permanente y su coeficiente debe ser positivo reflejando como la velocidad se mueve de una manera procíclica siendo así consistente con la hipótesis de la renta permanente (Friedman, 1957).

En primer lugar centramos nuestra atención más inmediata en el análisis de la velocidad de circulación en un período que consideramos nuestra aportación más innovadora a este estudio: los años 1850-1900. Estos nuevos datos exigen más análisis en la medida que desmienten hipótesis previas sobre su trayectoria, como las formuladas por Tortella (1983, 1994 y 1998), quien, a partir de algunos datos obtenidos para los años 1865-1894, pronosticaba la estabilidad de los niveles de V2 para antes de 1900.

La regresión para la ecuación (1) se ha realizado de 1851 a 1900 introduciendo una variable de tendencia que es significativa y una dummy para absorber el shock de la guerra de Cuba con su efecto posterior $(1898-1900)^{4}$.

CUADRO 4

LA FUNCIÓN DE LA VELOCIDAD DE CIRCULACIÓN DEL DINERO, 1851-1900

\begin{tabular}{|l|c|c|c|c|c|c|}
\hline & Constante & $\mathbf{Y}^{\mathbf{p}}$ & $\boldsymbol{i}$ & nalf & sof & def \\
\hline$(1)$ & $-42,902$ & 3,209 & 0,008 & 8,048 & 0,151 & 0,505 \\
& $(3,345) * *$ & $(0,221)^{* *}$ & $(0,004) *$ & $(0,714)^{* *}$ & $(0,275)$ & $(0,164)^{* *}$ \\
\hline
\end{tabular}

$\mathrm{DW}=1,11 \quad \mathrm{R}^{2}=0,9662 \quad \mathrm{~N}=50$

Fuente: Elaboración propia.

Notas: Coeficientes de las variables tradicionales e institucionales (errores estándar entre paréntesis).

* Estadísticamente significativo al 5 por ciento.

** Estadísticamente significativo al 1 por ciento.

${ }^{4}$ Se hicieron diversas estimaciones jugando con todas las variables disponibles, siendo ésta la que mejor explicaba el comportamiento de la velocidad. 
En una primera inspección de estos resultados nos sorprende que la renta permanente tenga signo positivo y que sea significativa al 1 por cien. Debemos recordar que el coeficiente previsto es $\left(1-\mathrm{A}_{1}\right)$, y ello implica que la elasticidad de la renta permanente para la demanda de dinero en el período considerado es negativa. En segundo lugar, el tipo de interés es positivo y significativo al 5 por cien y por tanto la elasticidad de la demanda de dinero es negativa según indica la teoría monetaria tradicional. Además, la inflación es positiva y significativa al 1 por cien. No parece el resultado esperable, pero debemos recordar que en un período de patrón metálico el signo correspondiente de la variación en los precios no es el mismo que en un período de moneda fiduciaria, sino precisamente el opuesto. Por lo que respecta a las variables institucionales, la monetización presenta signo positivo, contra lo que pudiéramos esperar, y es significativa al 1 por cien. En este caso nos enfrentamos a una paradoja característica de España donde, a lo largo de la segunda mitad del siglo XIX, por mucho que aumente la renta, la proporción de población activa no agraria — nuestra medida de la monetización- no disminuye. Quizá el grado de urbanización hubiese sido un indicador mejor, pero carecemos de una serie de urbanización con periodicidad anual. Por último, resta la variable que mide la sofisticación del sistema financiero. El coeficiente obtenido es positivo pero no es significativo, queriendo esto decir que esta variable no es representativa para este período. No nos debe extrañar, pues la sofisticación financiera se introduce para captar las etapas avanzadas del sistema financiero, y no para sus etapas iniciales, que son las que aparecen en la segunda mitad del siglo XIX. Podemos concluir entonces que de 1851 a 1900 la V española presenta una tendencia decreciente siendo su principal explicación una de las variables tradicionales —el tipo de interés-, mientras que las institucionales no parecen aportar capacidad explicativa.

Consideramos como siguiente objeto de estudio el comportamiento de la V en el largo plazo. Para este período decidimos centrarnos en los años de circulación de la peseta de 1868 a 1997, es decir, desde su creación hasta el último ejercicio completo antes de la transición de la peseta al euro ${ }^{5}$. El cuadro 5 presenta los resultados de la regresión en el muy largo plazo, 1868-1997, donde incluimos todas las variables de nuestra ecuación de la velocidad y además una variable dummy a partir de 1940 para corregir las fluctuaciones de la serie de monetización de los años cuarenta ${ }^{6}$.

5 Si se extiende el ejercicio desde 1850 en adelante, se pierde capacidad explicativa, lo que enfatiza la significación del cambio de régimen monetario que supone la creación de la peseta.

6 Esta dummy toma el valor de 1 a partir de 1940. 


\section{CUADRO 5}

LA FUNCIÓN DE LA VELOCIDAD DE CIRCULACIÓN DEL DINERO, 1868-1997

\begin{tabular}{|l|c|c|c|c|c|c|c|}
\hline & Constante & $\mathbf{Y}^{\mathbf{p}}$ & $\boldsymbol{i}$ & ciclo & nalf & sof & def \\
\hline$(2)$ & 3,320 & 0,417 & $-0,004$ & 0,743 & $-1,120$ & 0,377 & $-0,110$ \\
& $(0,549)^{* *}$ & $(0,057)^{* *}$ & $(0,003)$ & $(0,203)^{* * *}$ & $(0,205) * *$ & $(0,071)^{* *}$ & $(0,177)$ \\
\hline
\end{tabular}

$$
\mathrm{DW}=0,289 \quad \mathrm{R}^{2}=0,9590 \quad \mathrm{~N}=130
$$

Fuente: Elaboración propia. tesis).

Notas: Coeficientes de las variables tradicionales e institucionales (errores estándar entre parén-

* $\quad$ Estadísticamente significativo al 5 por ciento.
Estadísticamente significativo al 1 por ciento.

Esta regresión permite capturar la influencia significativa de la renta permanente y de las variables institucionales — población activa no agraria y sofisticación financiera-. Ello es indicio de que el comportamiento en forma de «U» de la velocidad a largo plazo se captura en una medida significativa. En cambio, las variables de interés y precios no salen significativas. Bien es verdad que el mezclar períodos con regímenes monetarios tan distintos puede erosionar enormemente su potencial explicativo.

Examinando los resultados obtenidos en la regresión (2) observamos cómo existe una severa autocorrelación entre los residuos de las variables que parece indicar que no existe una relación a largo plazo entre los componentes de la V. Obtenido este resultado, la manera más lógica de proceder es estimar el modelo en primeras diferencias. No obstante, los resultados de nuevo ofrecen signos negativos sobre el largo plazo, indicándonos la posibilidad de no encontrar una relación estable entre las variables de nuestro modelo pero sin poder llegar a ninguna conclusión definitiva ${ }^{7}$.

Los resultados obtenidos de las regresiones básicas no nos permiten afirmar si existe o no una relación estable de la velocidad en el largo plazo, lo que nos lleva a considerar el uso de técnicas econométricas más avanzadas. De esta forma y para continuar nuestro ejercicio y de manera similar a Siklos (1993) establecemos una relación de cointegración entre las variables que explican el comportamiento de la $\mathrm{V}$ en el largo plazo de 1850 a 2000. Sin embargo, la transición de la peseta al euro nos hace acotar nuestro estudio a 1997 al no encontrarse disponibles todas

\footnotetext{
7 Se corrieron diversas regresiones de modelos distintos pero los resultados obtenidos no fueron satisfactorios. Los resultados de las regresiones se pueden solicitar a los autores.
} 
las series necesarias ${ }^{8}$. Para ello y en primer lugar, se llevan a cabo los tests de raíz unitaria para todas las variables de nuestro estudio. Un test de raíz unitaria demostraría si nuestras variables son o no estacionarias y por tanto si existe una tendencia hacia un valor en el largo plazo. Los resultados obtenidos de los tests de raíz unitaria demuestran que, en España, de todas las variables que estamos considerando (las tradicionales y las institucionales) solamente dos de ellas no tienen una raíz unitaria: la tasa de inflación y el ciclo. Todas las demás son I (1) y, por tanto, no estacionarias ${ }^{9}$. Sin embargo, la preocupación primordial sobre la hipótesis del test de este paseo aleatorio yace en la escasez de suficiente poder para rechazarla y por ello este test puede proporcionarnos muy poca información en contra de la hipótesis alternativa relevante ${ }^{10}$. Para confirmar los resultados previos, se deben realizar unos tests de cointegración pues una combinación donde existen variables no estacionarias podría presentar una relación lineal en el largo plazo, o dicho de otro modo, podrían estar cointegradas.

En nuestro análisis, consideraremos dos relaciones de cointegración. La primera de ellas sólo incluye las variables tradicionales mientras que la segunda incluye tanto las tradicionales como las institucionales. La razón de esta división se encuentra en saber sí realmente las variables institutionales aportan algo nuevo al comportamiento de la velocidad o si por el contrario, no son esenciales para definir un modelo estable de la $\mathrm{V}$ en el largo plazo ${ }^{11}$.

Nuestra primera relación de cointegración es la siguiente:

$$
\log \mathrm{V}=\delta_{0}+\delta_{1} \log \mathrm{Y}^{\mathrm{p}}+\delta_{2} i+\mathrm{u}_{0} \quad \text { [Modelo 1] }
$$

donde estamos considerando las variables que hemos definido anteriormente ${ }^{12}$. Hay que notar, sin embargo, que si una variable tiene un orden de integración diferente al de las otras series, esta variable se omite, ya que es fundamental que todas las variables analizadas tengan el mismo orden de integración. De esta forma, las variables que no tienen una raíz unitaria, como son la tasa de inflación y el ciclo, quedan excluídas del análisis de cointegración.

8 Todos los análisis presentados a continuación se realizan usando el programa econométrico «JMulTi» ([Lütkepohl y Krätzig (eds.), 2004]).

9 Los resultados de los test de raíz unitaria se pueden solicitar a los autores.

10 Las series son estacionarias.

11 No hay datos de oferta monetaria para los años de la Guerra Civil (1936-1940). Este período, por tanto, se omite en la estimación usando variables dummies.

12 Para el análisis de cointegración consideramos la variable «tipo de interés» como su inversa, ya que la relación entre el logartimo de la velocidad y el tipo de interés parece ser más lineal al utilizar la relación inversa que los niveles (véase Anderson y Rasche, 2001). 
Para nuestro segundo test de cointegración incluimos tanto las variables tradicionales como las institucionales disponibles y siempre recordando que han de tener el mismo orden de integración. De esta forma el modelo 2 queda definido de la siguiente manera:

$\log \mathrm{V}=\beta_{0}+\beta_{1} \log \mathrm{Y}^{\mathrm{p}}+\beta_{2} i+\beta_{3} \log$ nalf $+\beta_{4} \log$ sof $+\mathrm{u}_{1} \quad$ [Modelo 2]

Para realizar los análisis de cointegración seguiremos el procedimiento de máxima probabilidad desarrollado por Johansen (Johansen, 1988; Johansen y Juselius, 1990) ${ }^{13}$. Como las variables analizadas pueden considerarse endógenas, es preferible realizar el test de cointegración a través de la metodología de VAR (Vector AutoRegression) donde el orden $k$ de los estadísticos se selecciona según el criterio de información de Akaike (Akaike Information Criteria, AIC) ${ }^{14}$. Los test estadísticos indican si se puede rechazar o no la hipótesis nula de, al menos, $r$ vectores de cointegración. Así por ejemplo diremos que el test evalua la hipótesis nula $r=0$ contra la alternativa $r \leq 1$. Nos estamos refiriendo al llamado «trace» test ${ }^{15}$. Existe, sin embargo, otro tipo de test para los análisis de cointegración, el llamado «maximum eigenvalue test.» El poder de los estadísticos de ambos tests es muy similar, incluso mayor para el «trace» test, cuando el período analizado es largo, y las conclusiones que se derivan son las mismas ${ }^{16}$. De ahí que centremos nuestra atención en los resultados proporcionados por el «trace» test. El cuadro 6 presenta los resultados del test de cointegración para los dos modelos estimados correspondientes al período de 1850 a $1997^{17}$.

El resultado encontrado para el modelo convencional de la V (modelo 1) indica que se puede rechazar la hipótesis nula de cero vectores de cointegración. Sin embargo, el test no puede rechazar la hipótesis de que el número de los vectores de cointegración es menor o igual a $1 \mathrm{y}$, por tanto, implica que $r=1$. Esto significa que existe un vector de cointegración o, lo que es lo mismo, una relación estable en el largo plazo

13 Para una descripción detallada de este procedimiento, veáse también Hafer y Jansen (1991).

14 El criterio de Schwartz para la selección de «lags» es otra opción pero tiende a ser más parsimonioso que el criterio Akaike. Optamos mejor por este último pues selecciona truncamientos relativamente largos reduciendo por tanto las posibilidades de ciertos tipos de errores de especificación.

15 El «trace» test examina que el rango de la matriz $\Pi$ de los vectores de cointegración es, al menos, $r$. Si el rango de la matriz $\Pi$ es $r<p$, esto implica que $\Pi=\alpha \beta^{\prime}$, donde $\alpha$ y $\beta$ son matrices $p \times r$. $\beta$ se interpretaría como la matriz de los parámetros de cointegración y $\alpha$ es la matriz de los parámetros de corrección de errores.

16 Veáse Lütkepohl, Saikkonen y Trenkler (2001).

17 Los tests de cointegración se realizan según la especificación básica sin incluir ninguna variable de tendencia por decisión propia ya que su consideración cuestiona la existencia de una relación económica fiable en el largo plazo. 
UN SIGLO Y MEDIO DE VELOCIDAD DE CIRCULACIÓN DEL DINERO EN ESPAÑA...

CUADRO 6

ANÁLISIS DE COINTEGRACIÓN: MÉTODO DE JOHANSEN

\begin{tabular}{|cc|cc|}
\hline Modelo 1 & & Modelo 2 & \\
\hline 0 & {$[3]$} & $\mathrm{r}$ & {$[3]$} \\
\hline 0 & $38,36^{*}$ & 0 & $169,15^{*}$ \\
$\leqslant 1$ & 16,61 & $\leqslant 1$ & $73,46^{*}$ \\
$\leqslant 2$ & 3,47 & $\leqslant 2$ & $41,08^{*}$ \\
& & $\leqslant 3$ & 18,61 \\
& & $\leqslant 4$ & 8,02 \\
\hline
\end{tabular}

Fuentes: Elaboración propia.

Notas: * Indica que la hipótesis nula de al menos $r$ vectores de cointegración no puede ser rechazada al 5 por ciento de significación.

Los valores críticos son de Johansen y Juselius (1990).

Todos los VAR incluyen una constante.

Entre paréntesis, el número de truncamientos (lags) según el criterio de Akaike (AIC).

entre la $\mathrm{V}$ y las dos variables tradicionales. A continuación realizamos un segundo test que repite el ejercicio del modelo 1 pero añadiendo las variables institucionales con la intención de corroborar que existe una relación de cointegración donde las variables institucionales aportan algo nuevo al sistema en el largo plazo. Los resultados obtenidos para el modelo 2 nos permiten rechazar la hipótesis de la existencia de dos o menos vectores de cointegración, $r \leq 2$. Con ello, podemos concluir que para este modelo existen tres vectores de cointegración, $r=3^{18}$. Una vez dicho esto debemos investigar si efectivamente las variables institucionales aumentan el efecto de estabilidad del modelo en el largo plazo. Es decir, nos interesa estudiar si estas variables añaden valor explicativo al comportamiento de la V siendo capaces de capturar el impacto de los cambios en el sistema financiero durante el período de tiempo considerado.

A continuación y para proporcionar un significado económico a los vectores de cointegración estimados estudiamos la elasticidad de la renta y del tipo de interés a la demanda de dinero. Para ello normalizamos los vectores de cointegración en la velocidad donde el coeficiente estimado es igual a 1. A través de esta normalización obtenemos los resultados de las elasticidades en el largo plazo.

Para el modelo 1, las elasticidades de la renta y del interés son ambas significativas pero esta última tiene el signo equivocado y la hipótesis

18 La presencia de cointegración indica que existe una relación estable en el largo plazo entre las variables consideradas. Cuanto mayor sea el número de vectores de cointegración, mayores son las posibilidades de encontrar relaciones lineales entre las variables para producir un sistema estacionario. 
nula de la elasticidad del tipo de interés igual a cero se rechaza. Basándonos en esta hipótesis, parece que el modelo básico de la velocidad dependiente solamente de las variables tradicionales está mal especificado, lo que nos hace centrar nuestra atención en el modelo 2, que considera las variables institucionales junto a las tradicionales.

En el modelo 2, los coeficientes de la renta permanente y del tipo de interés son significativamente diferentes de cero y presentan el signo correcto tal y como predice la teoría monetaria (cuadro 7). Sin embargo, para llegar a los resultados presentados en el cuadro 7 hay una serie de factores que tener en cuenta. El análisis estadístico nos sugiere que para obtener una representación parsimoniosa de los datos es necesario imponer una serie de restricciones en el modelo. Los datos sostienen restringir la variable que mide el proceso de monetización, nalf, ya que no ayuda a explicar el comportamiento de la V. Esto nos confirma el resultado obtenido en la regresión a corto plazo. La variable usada para medir el proceso de monetización en España en el largo plazo no parece ser un indicador muy adecuado pero, sin embargo, es el único con el que contamos. Por otra parte, los resultados de la regresión nos indican que la variable que mide la sofisticación financiera no parece ser muy estable al principio del período pero adquiere importancia - y significación- a partir de la década de $1950{ }^{19}$. De esta forma, llegamos a un modelo de velocidad que parece estar correctamente especificado y donde los coeficientes de las elasticidades en el largo plazo presentan los signos correctos tal y como predice la teoría monetaria y de acuerdo con las hipótesis tradicionales e institucionales de la velocidad.

Los valores estimados de las elasticidades de la renta y del tipo de interés son ambos positivos, siendo la primera menor que la unidad y de un valor inferior a la segunda. Una interpretación detallada de estos valores y siguiendo las indicaciones de la teoría monetaria tradicional

\section{CUADRO 7}

ELASTICIDADES DE LA RENTA Y DEL TIPO DE INTERÉS EN EL LARGO PLAZO

\begin{tabular}{|c|c|}
\hline Elasticidad de la renta & Semi-elasticidad del tipo de interés \\
\hline 0,498 & 1,615 \\
\hline
\end{tabular}

Fuentes: Elaboración propia.

Notas: Se presentan los resultados del vector de cointegración cuyo signo es consistente con la teoría monetaria después de la normalización y correspondiente al modelo $2^{1}$.

${ }^{1}$ Los resultados correspondientes al modelo 1 se pueden solicitar a los autores.

19 Los resultados obtenidos para la variable sof están disponibles y pueden solicitarse a los autores. 
nos lleva a afirmar que si la elasticidad de la renta permanente de la velocidad es mayor que cero y significativa, entonces la elasticidad de la renta permanente para la demanda de dinero es menor que la unidad. De la misma forma, si la semi-elasticidad del tipo de interés de la velocidad es positiva y significativa, su elasticidad en la demanda de dinero es, por tanto, negativa ${ }^{20}$.

Estos resultados sugieren la utilización de un modelo de vectores autorregresivos aumentado por los términos de corrección de errores para estudiar la existencia de una relación dinámica entre las variables consideradas. Un mecanismo de corrección de errores es, por tanto, un instrumento importante para asegurar los resultados obtenidos anteriormente en el análisis de cointegración. Así mismo, este mecanismo es capaz de determinar qué variables en el sistema son débilmente exógenas con respecto a las otras variables consideradas. En principio, los residuos de la regresión de equilibrio se usan para estimar los términos de la corrección de errores, los cuales son estadísticamente significativos cuando se estima el modelo de velocidad con restricciones. El resultado confirma que todas las variables son endógenas menos el tipo de interés y la sofisticación financiera que resultan ser débilmente exógenas. Además, podemos analizar el tamaño del término de corrección de error, donde encontramos que el ajuste hacia el equilibrio es lento, del orden aproximadamente del 0,12 ${ }^{21}$. Este resultado no es de sorprender, pues los cambios institucionales necesitan tiempo y sus efectos en la demanda de dinero son duraderos, tal y como postulan Bordo y Jonung (1987).

Por último, un análisis visual de las relaciones de cointegración para la ecuación de la velocidad nos ayuda a comprender mejor el significado de los términos de corrección de errores. El gráfico 5 presenta el coeficiente recursivo de corrección de error (vector alfa de la relación de cointegración) del modelo 2 para la ecuación de la velocidad con las dos bandas, inferior y superior, de los intervalos de confianza. Podemos observar cómo el coeficiente es relativamente estable cuando se consideran conjuntamente las variables tradicionales y las institucionales en el modelo 2. Sin embargo, este no es el caso cuando analizamos el modelo 1. El gráfico 6 demuestra cómo no existe estabilidad en el coeficiente de correción de error, alcanzando incluso valores no significativos. Por consiguiente, que el modelo de corrección de error no sea estable cuando las variables institucionales se omiten es una evidencia clara de su importancia a la hora de definir el comportamiento de la velocidad. El

${ }^{20}$ Recordemos que la variable usada en el análisis es la inversa del tipo de interés y, por tanto, el valor de la semi-elasticidad es $-1,615$.

21 Podemos comparar este resultado con el 0,07-0,08 por año obtenido por Bordo et al. (1997) para el Reino Unido y Suecia. El tamaño del término de corrección de error es pequeño y por tanto, el ajuste hacia el equilibrio es lento, tal y como sucede en el caso español. 
GRÁFICO 5

TÉRMINO DE CORRECCIÓN DE ERROR DEL MODELO 2

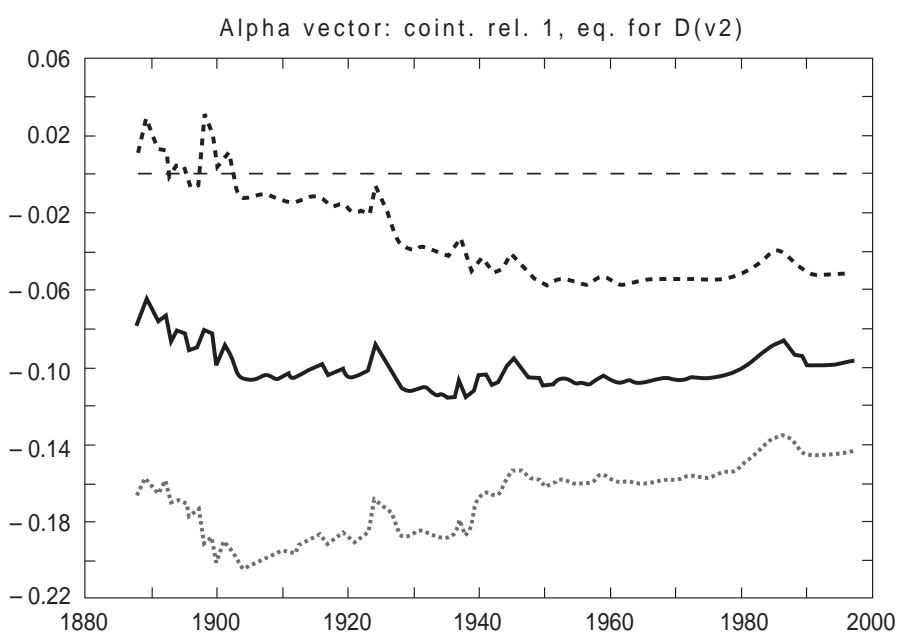

Fuentes: Elaboración propia.

GRÁFICO 6

TÉRMINO DE CORRECCIÓN DE ERROR DEL MODELO 1



Fuentes: Elaboración propia. 
hecho de encontrar una mayor estabilidad en el largo plazo para el modelo 2 que para el modelo 1 es una prueba de ello.

\section{CONCLUSIONES}

Recapitulando, nuestra nueva estimación de la V nos ha proporcionado algunas novedades. En primer lugar, en la segunda mitad del siglo XIX, la V cae desde niveles muy altos. La intensidad de la caída es irregular, alternándose fases de fuerte reducción con otras de notable estabilidad. Para el siglo Xx, nuestra mayor contribución es añadir los últimos veinte años. Con ellos llegamos a capturar la trayectoria en forma de «U» de la $\mathrm{V}$, que sólo es apreciable estadísticamente en torno a su punto de inflexión. En efecto, la perspectiva secular revela la importancia de la inflexión de la V en torno a 1973. Es el punto final de una larga caída y el inicio de una suave ascensión.

Hemos detectado que la $\mathrm{V}$ española se comporta de modo similar a la de algunos países para los que se disponen de buenos datos: Estados Unidos, Italia y Suecia. En el largo plazo, la V española se redujo con más lentitud que la sueca o la norteamericana, debido, fundamentalmente, a su menor ritmo de crecimiento económico. Su retraso en romper la tendencia descendente también parece derivarse del retraso en completar su maduración financiera. Observamos que los países agotan la reducción de la $\mathrm{V}$ cuando ésta alcanza, aproximadamente, una magnitud comparable al PIB monetario. Es también lo que sucede en España.

Las regresiones básicas - 1850 a 1900 y 1868 a 1997- no nos permiten llegar a ninguna conclusión sobre la existencia de una función estable de la velocidad, de ahí que nos adentremos en el análisis de cointegración y el mecanismo de correción de errores. Estas técnicas econométricas nos permiten examinar si las variables institucionales ayudan a explicar el comportamiento de la velocidad en España en el largo plazo. El modelo convencional de la $\mathrm{V}$ resulta no estar bien especificado y los resultados afirman su inestabilidad en el largo plazo. Por el contrario, el modelo que analiza las variables tradicionales junto a las institucionales aporta una nueva contribución, a pesar de las dificultades que algunas de estas variables presentan (i.e., la medida del proceso de monetización resulta ser inadecuada). La inclusión de las variables institucionales es importante para poder explicar el comportamiento de la $\mathrm{V}$ y confirmar así la existencia de una relación de equilibrio en el largo plazo. De esta forma, podemos concluir afirmando que realmente existe una función de velocidad estable para España que explica su patrón de comportamiento y, por tanto, su forma de U para el período de 1850 a 1997. 


\section{BIBLIOGRAFÍA}

ALCAIDE, J. (1976): «Una revisión urgente de la serie de renta nacional española en el siglo XX», en Datos básicos para la historia financiera de España, 1850-1975. Madrid: Instituto de Estudios Fiscales, vol.1, pp. 1127-1150.

ANDERSON, R. G., y RASche, R H. (2001): "The Remarkable Stability of Monetary Base Velocity in the United States, 1919-1999». Federal Reserve Bank of St. Louis, Working Paper 2001-008A.

BANCo de EsPaÑa (1980-2001): Boletín Estadístico. Madrid: Banco de España.

Bordo, M. D., y JonunG, L. (1981): «The Long-Run Behavior of the Income Velocity of Money in Five Advanced Countries, 1870-1975: An Institutional Approach». Economic Inquiry 19, pp. 96-116.

- (1987): The Long-run Behavior of the Velocity of Circulation. Cambridge: Cambridge University Press.

- (2004): Demand for Money. An Analysis of the Long-Run Behavior of the Velocity of Circulation. New Brunswick \& London: Transaction Publishers.

Bordo, M. D.; Jonung, L., y SiKlos, P. L. (1997): "Institutional Change and the Velocity of Money: A Century of Evidence». Economic Inquiry 35, pp. 710-724.

Bustelo, F., y Tortella, G. (1976): «Monetary Inflation in Spain, 1800-1970». Journal of European Economic History 5, pp. 141-150.

CAPIE, F. (2004): «Money and economic development in eighteenth-century England», en L. Prados de la Escosura (ed.), Exceptionalism and Industrialisation. Britain and its European Rivals, 1688-1815. Cambridge: Cambridge University Press, pp. 216-232.

CatalÁN, J.; Sudrià, C., y TiRAdo, D. A. (2001): «Peseta y protección: el debate historiográfico», en C. Sudrià y D. A. Tirado (eds.), Peseta y Protección. Comercio exterior, moneda y crecimiento económico en la España de la Restauración. Barcelona: Edicions Universitat de Barcelona, pp. 11-42.

Coll, S. (1989): «Estimación indirecta de una serie de renta nacional para España, 1874-1935. Primeros resultados», ponencia presentada al IV Congreso de la Asociación de Historia Económica. Alicante.

- (1992): «La renta nacional española, 1941-1953. Estimación de una serie a partir de variables monetarias». Santander: Universidad de Cantabria.

Comín, F. (1996): Historia de la Hacienda Pública II. Barcelona: Crítica.

Friedman, M. (1957): A Theory of the Consumption Function. Princeton: Princeton University Press.

Friedman, M., y Schwartz, A. J. (1963): A Monetary History of the United States. Princeton: Princeton University Press.

- (1982): Monetary Trends in the United States and the United Kingdom: Their Relation to Income, Prices, and Interest Rates, 1867-1975. Chicago: University of Chicago Press.

García Delgado, J. L., y Serrano, J. M. (dirs.), (2000): Del real al euro. Una historia de la peseta. Barcelona: «La Caixa», Servicio de Estudios.

García LóPeZ, J. R. (2001): "La letra de cambio, "papel moneda" del siglo XIX», en C. Sudrià y D. A. Tirado (eds.), Peseta y Protección. Comercio exterior, moneda y crecimiento económico en la España de la Restauración. Barcelona: Edicions Universitat de Barcelona, pp. 233-241.

GaRcía RUIZ, J. L. (1998): «Inflación y moneda en la España del siglo XX», ponencia presentada al Seminario sobre El legado del Modelo Castizo de desarrollo económico. Madrid: Real Academia de Ciencias Morales y Políticas. 
HAFER, R. W., y JANSEN, D. W. (1991): «The Demand for Money in the United States: Evidence from Cointegration Tests». Journal of Money, Credit and Banking 23, pp. 155-168.

JoHANSEN, S. (1988): «Statistical Analysis of Cointegrating Vectors». Journal of Economic Dynamics and Control 12, pp. 231-254.

Johansen, S., y Juselius, K. (1990): «Maximum Likelihood Estimation and Inference on Cointegration - With Applications to the Demand for Money». Oxford Bulletin of Economics and Statistics 52, pp. 169-210.

LÜtKePOHL, H., y KRÄTZIG, M. eds., (2004): Applied Time Series Econometrics. Cambridge: Cambridge University Press.

LÜTKePohl, H.; SAIKKONEN, P., y TRENKLER, C. (2001): «Maximum Eigenvalue versus Trace Tests for the Cointegrating Rank of a VAR Process». Econometrics Journal 4, pp. 287-310.

Maluquer de Motes, J. (1989): «Precios, salarios y beneficios», en A. Carreras (ed.), Estadísticas Históricas de España. Madrid: Fundación Banco Exterior, pp. 495-532.

- (2005): «Consumo y precios», en A. Carreras y X. Tafunell (eds.), Estadísticas Históricas de España. Madrid: Fundación BBVA, pp. 1247-1296.

Martín Aceña, P. (1985a): La cantidad de dinero en España, 1900-1935. Madrid: Banco de España.

- (1985b): «Desarrollo y modernización del sistema financiero, 1844-1935», en N. Sánchez Albornoz (ed.), La modernización económica de España 1830-1930. Madrid: Alianza Universidad, pp. 121-146.

- (1989): «El sistema financiero», en A. Carreras (ed.), Estadísticas Históricas de España. Madrid: Fundación Banco Exterior, pp. 363-394.

- (1990): «The Spanish Money Supply, 1874-1935». Journal of European Economic History 19, pp. 7-33.

- (2000): «The Spanish Monetary Experience, 1848-1914», en P. Martín Aceña y J. Reis (eds.), Monetary Standards in the Periphery. Paper, Silver and Gold, 18541933. London and New York: Macmillan \& St. Martin's Press, pp. 112-151.

Martín AcEÑA, P., y Pons, M. ${ }^{a}$ A. (2005): «Sistema monetario y financiero», en A. Carreras y X. Tafunell (eds.), Estadísticas Históricas de España. Madrid: Fundación BBVA, pp. 645-706.

MARTORELl, M. (2005): «Una guerra, dos pesetas», ponencia presentada a la sesión «La economía de la Guerra Civil, 1936-1939». Santiago de Compostela: VIII Congreso de la Asociación Española de Historia Económica.

MAYHEW, N. J. (1995): «Population, money supply, and the velocity of circulation in England, 1300-1700». Economic History Review, XLVIII, 2, pp. 238-257.

Prados de la Escosura, L. (2003): El progreso económico de España, 1850-2000. Madrid: Fundación BBVA.

SAINT-Marc, M. (1983): Histoire monétaire de la France, 1800-1980. Paris: Presses Universitaires de France.

SÁnCHEZ-Albornoz, N. (1975): Jalones en la modernización económica de España. Barcelona: Ariel.

SIKLOS, P. L. (1993): «Income Velocity and Institutional Change: Some New Time Series Evidence, 1870-1986». Journal of Money, Credit, and Banking 25, pp. 377-392.

SPINELLI, F., y FratianNi, M. (1991): Storia monetaria d'Italia. Milán: Mondadori.

SudRià, C., y PASCUAL, P. (1999): «Financing a railway mania: capital formation and the demand for money in Catalonia, 1840-66». Financial History Review 6, pp. 127-145. 
TAfunell, X. (2005): «Empresa y Bolsa», en A. Carreras y X. Tafunell (eds.), Estadísticas Históricas de España. Madrid: Fundación BBVA, pp. 707-834.

Tedde, P. (1999): El Banco de San Fernando (1829-1856). Madrid: Banco de España/Alianza.

Temin, P. (1969): The Jacksonian Economy. Norton.

Tortella, G. (1974): «Las magnitudes monetarias y sus determinantes», en G. Tortella (ed.), La banca española en la restauración. Madrid: Banco de España, vol. I, pp. 457-521.

- (1983): «National Income Estimation by Means of Monetary Variables, the Case of Spain, 1772-1972. Some Preliminary Results», en R. Fremdling y P. K. O'Brien (eds.), Productivity in the Economies of Europe. Stuttgart: Klett-Cotta, pp. 133-140.

- (1994): El desarrollo de la España contemporánea. Historia económica de los siglos XIX y XX. Madrid: Alianza Editorial.

- (1998): «La inflación española en el siglo XIX», ponencia presentada al Seminario sobre El legado del Modelo Castizo de desarrollo económico. Madrid: Real Academia de Ciencias Morales y Políticas.

- (2001): «¿Fue España diferente? La peseta en la época del patrón oro», en Banco de España (ed.), El Camino hacia el Euro. Madrid: Banco de España, pp. 63-84.

Velarde Fuertes, J. (2000): «La guerra de las dos pesetas, 1936-1939», en J. L. García Delgado y J. M. Serrano Sanz (dirs.), Del real al euro. Una historia de la peseta. Barcelona: «La Caixa», Servicio de Estudios, pp. 87-106. 


\section{APÉNDICE A}

\section{PRECEDENTES}

Nuestro estudio tiene como referencia los trabajos realizados previamente por otros investigadores económicos españoles sobre la velocidad de circulación (Coll, 1989, 1992; Martín Aceña, 1990; Tortella, 1983, 1994, 1998). Sin embargo, hay que destacar diferencias de orientación entre ellos a la hora de tratar la velocidad de circulación del dinero. El propósito de la mayoría de estos trabajos ha sido fundamentalmente utilizar las series de V para inferir información sobre otras variables económicas como, básicamente, el PIB real. En estos casos el supuesto de estabilidad de la V ha sido crucial.

Tortella (1983) calculó la primera serie histórica de la V española con el propósito de estimar el PIB español en el largo plazo. Para ello, utilizó la serie de precios que él había enlazado con Bustelo (Bustelo y Tortella, 1976), la serie de oferta monetaria que se deducía de los trabajos suyos (Tortella, 1974) y de los de Martín Aceña (entonces aún inéditos pero que se publicarían años después como Martín Aceña, 1985), y la serie de PIB de Alcaide (1976), correspondiente a 1900-1972, para obtener una V con la que después extrapolaba hacia el pasado, partiendo de un supuesto de estabilidad a largo plazo. Así estimaba valores probables del PIB español para mediados y finales del siglo XVIII. Obtuvo una serie anual de V de 1901 a 1972, completada con algunas observaciones para finales del siglo XIX (1865, 1879, 1882, 1888 y 1894). Esta primera serie de $\mathrm{V}$ se caracterizaba por una gran estabilidad con una tendencia decreciente en el siglo xx. Tortella la definió como una tendencia muy lenta y con un fuerte revés en los años 1901-1917. El resultado no despejaba la duda sobre la credibilidad de las series usadas para los años anteriores a 1900. Se hacía necesario una estimación de series temporales suficientemente largas y fiables para calcular una serie anual de $\mathrm{V}$ en el largo plazo.

Coll (1989) dio un importante paso adelante. Estimó una función de demanda de dinero para el período 1906-1935 (usando la estimación de renta nacional del Consejo de Economía Nacional) de la que obtuvo coeficientes y valores que aplicó a la estimación de la renta nacional de 1874 a 1935. El resultado, bastante convincente, le animó a realizar un nuevo intento orientado a la estimación del PIB del primer franquismo (19401954). En esta segunda ocasión, Coll (1992) partía también de la estimación de la función de demanda de dinero de 1954 a 1980, pero no quedó tan satisfecho como antes, principalmente porque observó que la proyección desde antes de la guerra civil era significativamente distinta 
a la retroproyección desde 1954-1980. Desgraciadamente para nuestra comunidad, sus dos intentos, el primero de ellos anticipado ya en el IV Congreso de la AHE, se quedaron en ensayos inéditos.

Cambiando por completo de enfoque, Martín Aceña (1990) entró de lleno en el cálculo e interpretación de la V para 1900-1935. En su caso el interés viró de la estimación de la renta nacional a la de la V. Sus resultados, muy razonables, no exploraban la riqueza de variación interanual que se encerraba en ellos. Probablemente, la convicción de que la base cuantitativa era frágil, impulsó a Martín Aceña a actuar con mucha precaución y a no explorar un área que podía resultar insatisfactoria. Tortella (1994) se animó a dar varios pasos adelante y a proponer una estimación y una interpretación detallada de la V para 1900-1980, y con detalle anual. Para ello combinó la serie de oferta monetaria (M2) de Martín Aceña (1989), la serie de precios que ya había usado con Bustelo (precios al por mayor) y el PIB de Alcaide. El resultado, afortunadamente para todos, no se aleja mucho del que hemos encontrado para el mismo período, pero sí que hay discrepancias significativas para varios subperiodos, principalmente 1910-1920. En cambio, nuestros resultados para la segunda mitad del siglo XIX se alejan mucho de los pronósticos que Tortella había formulado tanto en 1983 como en 1994. El último cálculo de la V lo encontramos en Tortella (1998) donde el autor, en su explicación del fenómeno inflacionista español (que prosigue para el siglo Xx en García Ruiz, 1998), calcula los valores de la V para los años 1865 y 1900. Los resultados obtenidos difieren de los nuestros, aunque levemente, dado que nosotros usamos la oferta monetaria M2 para calcular la V mientras que Tortella se basa en cálculos propios de la M1. Nuevamente, Tortella resalta en este trabajo la dificultad existente para calcular la V en España para los años anteriores a 1900 debido a la falta de series temporales fiables para este período. Aún así, se atreve a pronosticar que la tendencia al crecimiento de la V2 de 1900 a 1917 se debió haber originado hacia 1883, en ocasión de la desconexión de España del patrón oro.

Finalmente, en ocasión de los libros preparados en conmemoración de la desaparición de la peseta y en el homenaje a Sardá, algunos autores han mencionado el curso probable de la V (Catalán, Sudrià y Tirado, 2001; García Delgado y Serrano, eds., 2000; Tortella, 2001) en diversos períodos. Sin embargo, de todo ello no resulta ninguna versión coherente y sistematizada de la evolución de la V ni de sus factores determinantes. Esperamos haberla proporcionado en este artículo. 


\section{APÉNDICE B}

\section{FUENTES Y DATOS}

Para los ejercicios de estimación de los determinantes de la V y de la función de demanda de dinero hemos recurrido a diversas variables, cuyas fuentes describimos a continuación.

La renta permanente ha sido calculada a partir del PIB real per cápita a precios de mercado (Prados de la Escosura, 2003). Hemos supuesto que el valor de 1850 era la renta permanente inicial. Para los años siguientes hemos aplicado los parámetros estimados en su día por Friedman y Schwartz (1963), utilizada por Bordo y Jonung (1987) y Siklos (1993) ${ }^{22}$.

La serie de tipo de interés se ha construido combinando la serie de tasas de rendimiento interno de la deuda pública española (perpetua al 4 por cien) de 1850 a 1935, y la de la deuda pública a tres años de 1982 en adelante, ambas procedentes de Tafunell (2005, cuadro 35). Los huecos se han rellenado recurriendo a tres fuentes diversas. De 1960 a 1981, ambos incluidos, se trata de las tasas de rendimiento interno de las obligaciones del sector privado, obtenidas de Tafunell (2005, cuadro 35). De 1942 a 1960 hemos recurrido al tipo de descuento comercial del Banco de España, proporcionado por Martín Aceña (1989, cuadro 9.8). A la vista de la apreciable similitud de ambas series en los años de enlace, nos hemos abstenido de cualquier otra manipulación estadística. Para 19351942 hemos proyectado los valores mediante el uso de los determinantes más importantes que afectan a esta variable (i.e., el PIB y la oferta de dinero) ${ }^{23}$.

La serie de inflación esperada ha sido estimada a partir de las primeras diferencias del deflactor del PIB a precios de mercado (Prados de la Escosura, 2003, cuadro A.13.6).

La proporción de población empleada en los sectores no agrarios procede de Leandro Prados de la Escosura (2003, cuadro A.11.4).

La sofisticación financiera consiste en la proporción entre dinero legal y oferta monetaria. El dinero legal (moneda metálica y billetes en circulación) procede de Martín Aceña (1989) y Martín Aceña y Pons (2005). Las fuentes de la oferta monetaria han sido explicadas en el apartado 2. Para el período 1850-1875 debe advertirse, sin embargo, que es difícil distinguir el stock de oro y plata del oro y plata en circulación

22 El algoritmo usado es $\mathrm{y}_{\mathrm{t}}^{\mathrm{p}}=\mathrm{A}\left(0.32968 \mathrm{y}_{\mathrm{t}}+0.67032 \mathrm{y}_{\mathrm{t}-1}^{\mathrm{p}}\right)$, siendo A el crecimiento de la renta real per cápita en el largo plazo $(\mathrm{A}=1.01530)$, véase Siklos, 1993.

${ }^{23}$ Los valores obtenidos para el tipo de interés (en porcentajes) son: 1936, 4.75; 1937, 5.40; 1938, 6.90; 1939, 6.45; 1940, 5.85; 1941, 4.66. 
(véase Martín Aceña, 1989, cuadro 9.1). Para obviar esta dificultad hemos procedido a calcular el dinero legal como la diferencia entre la oferta monetaria - bien fundamentada en la literatura- y las cuentas corrientes en el Banco de España y en la banca privada - sobre las que no hay dudas-. Para todo ello hemos recurrido a Martín Aceña (1989, cuadros 9.2 y 9.4), de acuerdo con la pauta que él establece en Martín Aceña (2000, cuadro 3.3).

A continuación se presentan las series estadísticas empleadas cuando son elaboraciones propias.

CUADRO B.1

LOS DETERMINANTES DE LA VELOCIDAD DE CIRCULACIÓN DEL DINERO, 1850-2000

\begin{tabular}{|c|c|c|c|c|}
\hline Año & $\begin{array}{c}\text { Renta } \\
\text { permanente }\end{array}$ & $\begin{array}{c}\text { Tipo } \\
\text { de interés }\end{array}$ & $\begin{array}{c}\text { Tasa } \\
\text { de inflación }\end{array}$ & $\begin{array}{c}\text { Sofisticación } \\
\text { financiera }\end{array}$ \\
\hline $1850 \ldots$ & 137,0 & 9,4 & & 1,00 \\
\hline $1851 \ldots \ldots \ldots$ & 139,5 & 8,4 & 0,7 & 1,00 \\
\hline 1852. & 143,1 & 6,9 & $-4,0$ & 1,00 \\
\hline $1853 \ldots$. & 145,7 & 6,9 & 17,6 & 1,00 \\
\hline $1854 \ldots \ldots \ldots \ldots \ldots \ldots \ldots \ldots$ & 148,1 & 8,5 & 2,2 & 1,00 \\
\hline $1855 \ldots \ldots \ldots \ldots \ldots \ldots \ldots$ & 151,9 & 9,2 & 2,3 & 1,00 \\
\hline $1856 \ldots \ldots \ldots \ldots \ldots \ldots \ldots \ldots$ & 152,8 & 7,4 & 1,5 & 0,97 \\
\hline $1857 \ldots \ldots \ldots \ldots \ldots \ldots \ldots$ & 152,1 & 7,7 & $-2,8$ & 0,96 \\
\hline $1858 \ldots \ldots \ldots \ldots \ldots \ldots$ & 152,5 & 7,5 & $-4,5$ & 0,95 \\
\hline 1859 & 154,8 & 7,3 & 1,7 & 0,94 \\
\hline $1860 \ldots \ldots \ldots \ldots \ldots \ldots$ & 157,9 & 6,4 & 2,7 & 0,93 \\
\hline $1861 \ldots \ldots \ldots \ldots \ldots \ldots \ldots$ & 160,5 & 6,3 & 0,7 & 0,95 \\
\hline $1862 \ldots$ & 162,5 & 6,0 & 2,2 & 0,94 \\
\hline 1863. & 164,9 & 5,8 & 2,9 & 0,96 \\
\hline $1864 \ldots$ & 166,4 & 6,1 & $-0,1$ & 0,97 \\
\hline $1865 \ldots$ & 165,5 & 7,3 & $-3,0$ & 0,97 \\
\hline $1866 \ldots \ldots \ldots \ldots \ldots \ldots$ & 167,5 & 8,8 & 1,9 & 0,98 \\
\hline $1867 \ldots \ldots \ldots \ldots \ldots$ & 168,5 & 8,9 & 7,0 & 0,96 \\
\hline $1868 \ldots \ldots \ldots \ldots \ldots \ldots$ & 163,6 & 8,7 & $-5,3$ & 0,97 \\
\hline 1869 & 161,5 & 11,3 & $-10,6$ & 0,98 \\
\hline $1870 \ldots$ & 161,3 & 11,7 & 5,7 & 1,00 \\
\hline $1871 \ldots$ & 164,9 & 10,5 & $-0,4$ & 1,05 \\
\hline $1872 .$. & 174,9 & 10,8 & 0,8 & 1,10 \\
\hline $1873 \ldots$ & 186,9 & 11,7 & $-1,9$ & 1,16 \\
\hline $1874 \ldots$ & 189,2 & 15,6 & 6,4 & 1,20 \\
\hline $1875 \ldots$ & 192,4 & 12,2 & $-3,6$ & 0,94 \\
\hline $1876 \ldots$ & 195,5 & 15,0 & 1,4 & 0,91 \\
\hline $1877 \ldots \ldots$ & 204,0 & 8,4 & 0,1 & 0,91 \\
\hline $1878 \ldots \ldots \ldots \ldots \ldots \ldots \ldots$ & 207,6 & 6,9 & 1,6 & 0,89 \\
\hline 1879 & 205,9 & 6,4 & 2,8 & 0,88 \\
\hline $1880 \ldots \ldots \ldots$ & 210,1 & 5,2 & $-2,5$ & 0,84 \\
\hline $1881 \ldots \ldots \ldots \ldots \ldots \ldots$ & 214,4 & 3,8 & 2,5 & 0,77 \\
\hline $1882 \ldots$ & 217,8 & 4,2 & 4,4 & 0,84 \\
\hline $1883 \ldots$ & 221,4 & 6,2 & $-0,3$ & 0,84 \\
\hline $1884 \ldots \ldots \ldots \ldots \ldots \ldots$ & 223,6 & 6,4 & $-4,2$ & 0,81 \\
\hline
\end{tabular}


CUADRO B.1 (Cont.)

\begin{tabular}{|c|c|c|c|c|}
\hline Año & $\begin{array}{c}\text { Renta } \\
\text { permanente }\end{array}$ & $\begin{array}{c}\text { Tipo } \\
\text { de interés }\end{array}$ & $\begin{array}{c}\text { Tasa } \\
\text { de inflación }\end{array}$ & $\begin{array}{c}\text { Sofisticación } \\
\text { financiera }\end{array}$ \\
\hline $1885 .$. & 222,8 & 6,6 & 1,4 & 0,80 \\
\hline $1886 \ldots \ldots \ldots \ldots \ldots \ldots$ & 220,3 & 6,4 & 4,0 & 0,77 \\
\hline $1887 \ldots \ldots \ldots \ldots \ldots \ldots$ & 217,4 & 5,9 & $-7,3$ & 0,76 \\
\hline $1888 \ldots \ldots \ldots \ldots \ldots \ldots \ldots$ & 217,7 & 5,5 & 0,9 & 0,76 \\
\hline $1889 \ldots \ldots \ldots \ldots \ldots \ldots \ldots$ & 217,5 & 5,2 & $-7,8$ & 0,73 \\
\hline $1890 \ldots \ldots \ldots \ldots \ldots \ldots \ldots \ldots$ & 217,1 & 5,1 & 1,0 & 0,73 \\
\hline $1891 \ldots \ldots \ldots \ldots \ldots \ldots \ldots \ldots$ & 218,0 & 5,1 & $-0,1$ & 0,72 \\
\hline $1892 \ldots$ & 223,6 & 5,7 & $-7,6$ & $0,7 \overline{5}$ \\
\hline $1893 \ldots \ldots \ldots \ldots \ldots \ldots \ldots$ & 224,5 & 5,6 & $-0,1$ & 0,75 \\
\hline $1894 \ldots$ & 225,5 & 5,5 & $-2,9$ & 0,76 \\
\hline $1895 \ldots$ & 225,3 & 5,6 & 1,9 & 0,74 \\
\hline $1896 \ldots$. & 219,1 & 6,1 & 3,8 & 0,73 \\
\hline $1897 \ldots$. & 217,9 & 6,0 & 3,7 & 0,72 \\
\hline $1898 \ldots$ & 222,1 & 7,2 & $-0,2$ & 0,69 \\
\hline $1899 \ldots \ldots \ldots \ldots \ldots \ldots$ & 225,8 & 6,1 & $-0,8$ & 0,69 \\
\hline $1900 \ldots \ldots \ldots \ldots \ldots \ldots \ldots$ & 229,6 & 4,5 & 3,4 & 0,71 \\
\hline $1901 \ldots \ldots \ldots \ldots \ldots \ldots \ldots \ldots$ & 237,1 & 4,5 & $-1,4$ & 0,70 \\
\hline $1902 \ldots \ldots \ldots \ldots \ldots \ldots \ldots$ & 239,3 & 4,4 & $-0,4$ & 0,71 \\
\hline ….......... & 240,6 & 4,2 & 6,6 & 0,69 \\
\hline $1904 \ldots$. & 240,7 & 4,2 & 6,0 & 0,68 \\
\hline $1905 \ldots \ldots \ldots \ldots \ldots \ldots$ & 239,4 & 4,1 & $-2,4$ & 0,67 \\
\hline $1906 \ldots . .$. & 241,6 & 4,0 & $-3,3$ & 0,65 \\
\hline $1907 \ldots$ & 245,1 & 3,9 & 1,2 & 0,65 \\
\hline $1908 \ldots$ & 250,0 & 3,9 & $-4,3$ & 0,64 \\
\hline $1909 \ldots$ & 254,2 & 3,7 & 0,4 & 0,63 \\
\hline $1910 \ldots \ldots \ldots \ldots \ldots \ldots$ & 253,6 & 3,8 & $-1,4$ & 0,63 \\
\hline $1911 \ldots \ldots \ldots \ldots \ldots \ldots \ldots$ & 258,3 & 3,8 & 1,3 & 0,63 \\
\hline $1912 \ldots \ldots \ldots \ldots \ldots \ldots \ldots \ldots$ & 260,3 & 3,8 & 2,7 & 0,63 \\
\hline $1913 \ldots \ldots \ldots \ldots \ldots \ldots \ldots \ldots$ & 264,6 & 4,0 & 2,6 & 0,63 \\
\hline $1914 \ldots \ldots \ldots \ldots \ldots \ldots \ldots \ldots$ & 265,7 & 4,2 & 1,6 & 0,63 \\
\hline $1915 \ldots \ldots \ldots \ldots \ldots \ldots$ & 267,2 & 4,5 & 9,5 & 0,59 \\
\hline $1916 \ldots \ldots \ldots \ldots \ldots \ldots$ & 271,7 & 4,3 & 10,5 & 0,58 \\
\hline $1917 \ldots$. & 273,1 & 4,3 & 10,4 & 0,55 \\
\hline $1918 \ldots$ & 272,8 & 4,1 & 22,6 & 0,51 \\
\hline $1919 \ldots \ldots \ldots \ldots \ldots \ldots$ & 272,5 & 4,2 & 6,6 & 0,51 \\
\hline $1920 \ldots \ldots \ldots \ldots \ldots \ldots$ & 278,0 & 4,4 & 9,9 & 0,50 \\
\hline $1921 \ldots \ldots \ldots \ldots \ldots \ldots$ & 283,3 & 4,7 & $-10,8$ & 0,49 \\
\hline $1922 \ldots \ldots \ldots \ldots \ldots \ldots \ldots$ & 289,9 & 4,6 & $-2,7$ & 0,43 \\
\hline 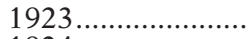 & 294,6 & 4,5 & $-1,6$ & 0,43 \\
\hline $1924 \ldots \ldots \ldots \ldots \ldots \ldots \ldots \ldots$ & 299,6 & 4,6 & 5,8 & 0,45 \\
\hline $1925 \ldots \ldots \ldots \ldots \ldots \ldots$ & 308,1 & 4,6 & 0,3 & 0,44 \\
\hline $1926 \ldots$ & 312,4 & 4,7 & $-2,2$ & 0,43 \\
\hline $1927 \ldots$ & 323,1 & 4,6 & $-1,3$ & 0,39 \\
\hline $1928 \ldots$ & 329,8 & 4,3 & $-3,3$ & 0,38 \\
\hline $1929 \ldots$ & 340,9 & 4,3 & 1,1 & 0,36 \\
\hline $1930 \ldots$ & 343,4 & 4,5 & 4,1 & 0,36 \\
\hline $1931 \ldots \ldots \ldots \ldots \ldots \ldots$ & 341,2 & 5,5 & 0,8 & 0,40 \\
\hline $1932 \ldots \ldots \ldots \ldots \ldots$ & 341,0 & 5,0 & $-2,5$ & 0,38 \\
\hline $1933 \ldots \ldots \ldots \ldots \ldots \ldots$ & 337,8 & 4,8 & $-2,6$ & 0,37 \\
\hline $1934 \ldots \ldots \ldots \ldots \ldots \ldots$ & 338,5 & 4,6 & 6,4 & 0,35 \\
\hline $1935 \ldots \ldots \ldots \ldots \ldots \ldots \ldots$ & 340,2 & 4,2 & 1,1 & 0,34 \\
\hline $1936 \ldots$ & 316,1 & 4,7 & 4,8 & $\ldots$ \\
\hline
\end{tabular}


CUADRO B.1 (Cont.)

\begin{tabular}{|c|c|c|c|c|}
\hline Año & $\begin{array}{c}\text { Renta } \\
\text { permanente }\end{array}$ & $\begin{array}{c}\text { Tipo } \\
\text { de interés }\end{array}$ & $\begin{array}{c}\text { Tasa } \\
\text { de inflación }\end{array}$ & $\begin{array}{c}\text { Sofisticación } \\
\text { financiera }\end{array}$ \\
\hline $1937 \ldots$ & 292,0 & 5,4 & 14,1 & $\cdots$ \\
\hline $1938 \ldots \ldots \ldots \ldots$ & 274,8 & 6,9 & 13,7 & $\ldots$ \\
\hline $1939 \ldots \ldots \ldots \ldots$ & 268,4 & 6,4 & 7,0 & $\ldots$ \\
\hline 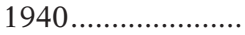 & 271,1 & 5,8 & 14,1 & \\
\hline $1941 \ldots \ldots \ldots \ldots \ldots \ldots$ & 270,8 & 4,7 & 10,0 & 0,38 \\
\hline $1942 \ldots \ldots \ldots \ldots \ldots \ldots \ldots$ & 274,7 & 4,0 & 13,7 & 0,41 \\
\hline $1943 \ldots \ldots \ldots \ldots \ldots \ldots \ldots$ & 279,9 & 4,0 & 2,9 & 0,40 \\
\hline $1944 \ldots \ldots \ldots \ldots \ldots \ldots \ldots \ldots \ldots$ & 287,1 & 4,0 & 10,9 & 0,38 \\
\hline 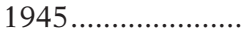 & 284,7 & 4,0 & 7,4 & 0,36 \\
\hline $1946 \ldots \ldots \ldots \ldots \ldots \ldots \ldots \ldots$ & 286,4 & 4,0 & 21,5 & 0,35 \\
\hline $1947 \ldots .$. & 288,3 & 4,5 & 13,9 & 0,35 \\
\hline $1948 \ldots$ & 289,1 & 4,5 & 7,6 & 0,33 \\
\hline $1949 \ldots \ldots$ & 288,4 & 4,0 & 7,2 & 0,31 \\
\hline $1950 \ldots$. & 290,0 & 4,0 & 20,9 & 0,31 \\
\hline $1951 \ldots \ldots \ldots \ldots \ldots$ & 299,6 & 4,0 & 16,6 & 0,31 \\
\hline $1952 \ldots \ldots \ldots \ldots \ldots \ldots \ldots \ldots$ & 313,5 & 4,0 & 1,3 & 0,28 \\
\hline $1953 \ldots \ldots \ldots \ldots \ldots \ldots \ldots$ & 321,7 & 4,0 & 8,1 & 0,26 \\
\hline $1954 \ldots \ldots \ldots \ldots \ldots \ldots \ldots$ & 334,5 & 3,8 & 8,0 & 0,25 \\
\hline $1955 \ldots \ldots \ldots \ldots \ldots \ldots$ & 346,8 & 3,8 & 4,6 & 0,23 \\
\hline 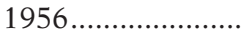 & 363,8 & 4,3 & 14,4 & 0,23 \\
\hline 1957 .................... & 378,4 & 5,0 & 13,0 & 0,24 \\
\hline $1958 \ldots .$. & 392,8 & 5,0 & 11,8 & 0,23 \\
\hline $1959 \ldots$ & 398,4 & 6,3 & 5,3 & 0,23 \\
\hline $1960 \ldots \ldots \ldots \ldots \ldots$ & 403,2 & 6,2 & 2,2 & 0,21 \\
\hline $1961 \ldots \ldots \ldots \ldots \ldots \ldots$ & 422,0 & 5,7 & 1,8 & 0,20 \\
\hline $1962 \ldots \ldots \ldots \ldots \ldots \ldots$ & 450,0 & 5,2 & 5,4 & 0,20 \\
\hline $1963 \ldots \ldots \ldots \ldots \ldots \ldots \ldots$ & 483,7 & 5,2 & 7,7 & 0,20 \\
\hline $1964 \ldots \ldots \ldots \ldots \ldots \ldots \ldots \ldots$ & 521,2 & 5,7 & 7,0 & 0,19 \\
\hline $1965 \ldots \ldots \ldots \ldots \ldots \ldots \ldots \ldots$ & 557,1 & 6,0 & 9,0 & 0,19 \\
\hline $1966 \ldots \ldots \ldots \ldots \ldots \ldots \ldots$ & 594,3 & 6,8 & 7,3 & 0,19 \\
\hline 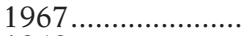 & 631,2 & 7,2 & 6,2 & 0,18 \\
\hline $1968 \ldots \ldots \ldots \ldots \ldots \ldots$ & 667,1 & 7,3 & 4,9 & 0,17 \\
\hline 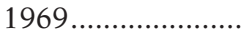 & 710,5 & 7,7 & 3,5 & 0,16 \\
\hline $1970 \ldots \ldots \ldots \ldots \ldots$ & 752,1 & 8,9 & 6,6 & 0,15 \\
\hline $1971 \ldots \ldots \ldots \ldots \ldots \ldots \ldots$ & 793,1 & 9,4 & 8,1 & 0,13 \\
\hline $1972 \ldots \ldots \ldots \ldots \ldots \ldots$ & 841,0 & 8,7 & 7,9 & 0,12 \\
\hline 1973 …………... & 897,3 & 8,7 & 11,6 & 0,12 \\
\hline 1974 & 956,1 & 10,8 & 14,4 & 0,11 \\
\hline $1975 \ldots \ldots \ldots \ldots \ldots \ldots \ldots$ & 1004,3 & 11,0 & 15,0 & 0,11 \\
\hline $1976 \ldots \ldots \ldots \ldots \ldots \ldots \ldots$ & 1047,8 & 10,3 & 15,6 & 0,11 \\
\hline $1977 \ldots \ldots \ldots \ldots \ldots \ldots \ldots$ & 1087,3 & 10,2 & 23,3 & 0,12 \\
\hline $1978 \ldots \ldots \ldots \ldots$ & 1122,4 & 11,4 & 20,1 & 0,12 \\
\hline $1979 \ldots$ & 1148,5 & 14,2 & 15,0 & 0,11 \\
\hline $1980 \ldots$. & 1172,4 & 15,2 & 11,1 & 0,11 \\
\hline $1981 \ldots$ & 1188,5 & 15,5 & 12,7 & 0,10 \\
\hline $1982 \ldots$ & 1204,3 & 16,0 & 14,0 & 0,10 \\
\hline $1983 \ldots \ldots \ldots \ldots \ldots$ & 1223,2 & 16,9 & 12,1 & 0,10 \\
\hline $1984 \ldots \ldots \ldots+\ldots$ & 1240,2 & 16,5 & 12,0 & 0,10 \\
\hline $1985 \ldots \ldots \ldots$ & 1258,6 & 13,4 & 7,4 & 0,11 \\
\hline $1986 \ldots \ldots \ldots \ldots \ldots \ldots$ & 1283,5 & 11,4 & 10,7 & 0,12 \\
\hline $1987 \ldots \ldots \ldots \ldots \ldots \ldots \ldots \ldots$ & 1323,6 & 13,6 & 5,4 & 0,13 \\
\hline $1988 \ldots$ & 1374,5 & 11,8 & 5,4 & 0,13 \\
\hline
\end{tabular}


CUADRO B.1 (Cont.)

\begin{tabular}{|c|c|c|c|c|}
\hline Año & $\begin{array}{c}\text { Renta } \\
\text { permanente }\end{array}$ & $\begin{array}{l}\text { Tipo } \\
\text { de interés }\end{array}$ & $\begin{array}{c}\text { Tasa } \\
\text { de inflación }\end{array}$ & $\begin{array}{c}\text { Sofisticación } \\
\text { financiera }\end{array}$ \\
\hline $1989 .$. & 1433,1 & 13,6 & 6,5 & 0,14 \\
\hline $1990 \ldots \ldots \ldots \ldots \ldots \ldots \ldots$ & 1494,4 & 14,6 & 7,1 & 0,14 \\
\hline $1991 \ldots$ & 1549,6 & 12,5 & 6,8 & 0,15 \\
\hline $1992 \ldots$ & 1597,6 & 12,6 & 6,6 & 0,15 \\
\hline 1993.. & 1630,5 & 10,1 & 4,7 & 0,15 \\
\hline $1994 \ldots \ldots \ldots \ldots \ldots \ldots \ldots$ & 1665,8 & 9,3 & 4,0 & 0,15 \\
\hline $1995 \ldots \ldots \ldots \ldots \ldots \ldots \ldots$ & 1706,9 & 10,9 & 4,6 & 0,15 \\
\hline $1996 \ldots \ldots \ldots \ldots \ldots \ldots \ldots$ & 1747,3 & 7,7 & 3,5 & 0,15 \\
\hline $1997 \ldots \ldots \ldots \ldots \ldots \ldots \ldots$ & 1795,7 & 5,3 & 2,2 & 0,16 \\
\hline 1998_.................. & 1852,1 & 4,1 & 2,3 & $\ldots$ \\
\hline 1999 & 1913,3 & 3,7 & 2,9 & $\ldots$ \\
\hline 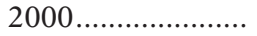 & 1980,8 & 5,1 & 3,5 & $\ldots$ \\
\hline
\end{tabular}

Fuentes: Ver apéndice B. 
